\title{
OPEN An in silico deep learning approach to multi-epitope vaccine design: a SARS-CoV-2 case study
}

\author{
Zikun Yang, Paul Bogdan ${ }^{\bowtie}$ \& Shahin Nazarian
}

The rampant spread of COVID-19, an infectious disease caused by SARS-CoV-2, all over the world has led to over millions of deaths, and devastated the social, financial and political entities around the world. Without an existing effective medical therapy, vaccines are urgently needed to avoid the spread of this disease. In this study, we propose an in silico deep learning approach for prediction and design of a multi-epitope vaccine (DeepVacPred). By combining the in silico immunoinformatics and deep neural network strategies, the DeepVacPred computational framework directly predicts 26 potential vaccine subunits from the available SARS-CoV-2 spike protein sequence. We further use in silico methods to investigate the linear B-cell epitopes, Cytotoxic T Lymphocytes (CTL) epitopes, Helper T Lymphocytes (HTL) epitopes in the 26 subunit candidates and identify the best 11 of them to construct a multi-epitope vaccine for SARS-CoV-2 virus. The human population coverage, antigenicity, allergenicity, toxicity, physicochemical properties and secondary structure of the designed vaccine are evaluated via state-of-the-art bioinformatic approaches, showing good quality of the designed vaccine. The $3 \mathrm{D}$ structure of the designed vaccine is predicted, refined and validated by in silico tools. Finally, we optimize and insert the codon sequence into a plasmid to ensure the cloning and expression efficiency. In conclusion, this proposed artificial intelligence (Al) based vaccine discovery framework accelerates the vaccine design process and constructs a 694aa multi-epitope vaccine containing 16 B-cell epitopes, $82 \mathrm{CTL}$ epitopes and $89 \mathrm{HTL}$ epitopes, which is promising to fight the SARS-CoV-2 viral infection and can be further evaluated in clinical studies. Moreover, we trace the RNA mutations of the SARS-CoV-2 and ensure that the designed vaccine can tackle the recent RNA mutations of the virus.

Coronavirus disease 2019 (COVID-19) is an infectious disease caused by severe acute respiratory syndrome coronavirus 2 (SARS-CoV-2) ${ }^{1,2}$. First detected in December 2019 in Wuhan, the virus has spread globally, with basic reproduction number (R0) reaching $5.7^{3}$, millions of deaths, and unprecedented financial, social and political impacts all over the world ${ }^{4}$. Efficacious vaccines are therefore desperately needed ${ }^{5}$. The main clinical features of the COVID-19 are fever, cough and myalgia or fatigue ${ }^{6}$; the virus has caused clusters of severe respiratory illness similar to severe acute respiratory syndrome coronavirus and is associated with ICU (Intensive Care Unit) admission and high mortality rates ${ }^{7}$.

Currently, without a single specific antiviral therapy for SARS-CoV-2, the control methods of the COVID-19 are early diagnosis, reporting, isolation, supportive treatments, and timely publishing epidemic information with only limited impact on the coronavirus ${ }^{8,9}$. Researchers have proposed several approaches to develop vaccines for the SARS-CoV- $2^{10}$. Traditional process of vaccine design is based on growing pathogens, which represents a very time-consuming process of isolating, inactivating and injecting the virus that causes the disease $\mathrm{e}^{11,12}$. Such process usually takes more than a year to result in efficacious vaccines and hence contributes very little to avoid the current spread of the disease $\mathrm{e}^{13,14}$. Recently, researchers have worked on constructing multi-epitope vaccines by in silico methods based on immunoinformatics without the need to grow pathogens to accelerate the vaccine design process ${ }^{15-17}$. Multi-epitope vaccines are constructed by multiple virus protein fragments rich in overlapping epitopes. They contain the vital part of the virus to elicit either a cellular or a humoral immune response and they reduce unwanted components that can trigger adverse effects ${ }^{18}$. Multi-epitope vaccines can be powerful for fighting viral infections, providing excellent vaccine candidates for clinical trials. The genome sequencing of the SARS-CoV-2 is completed ${ }^{8}$ and researchers have studied the details in the SARS-CoV-2 proteins ${ }^{19}$. Coronavirus is studded on its exterior with spike proteins, which are key components to infect and attack human cells ${ }^{20}$. The 
A
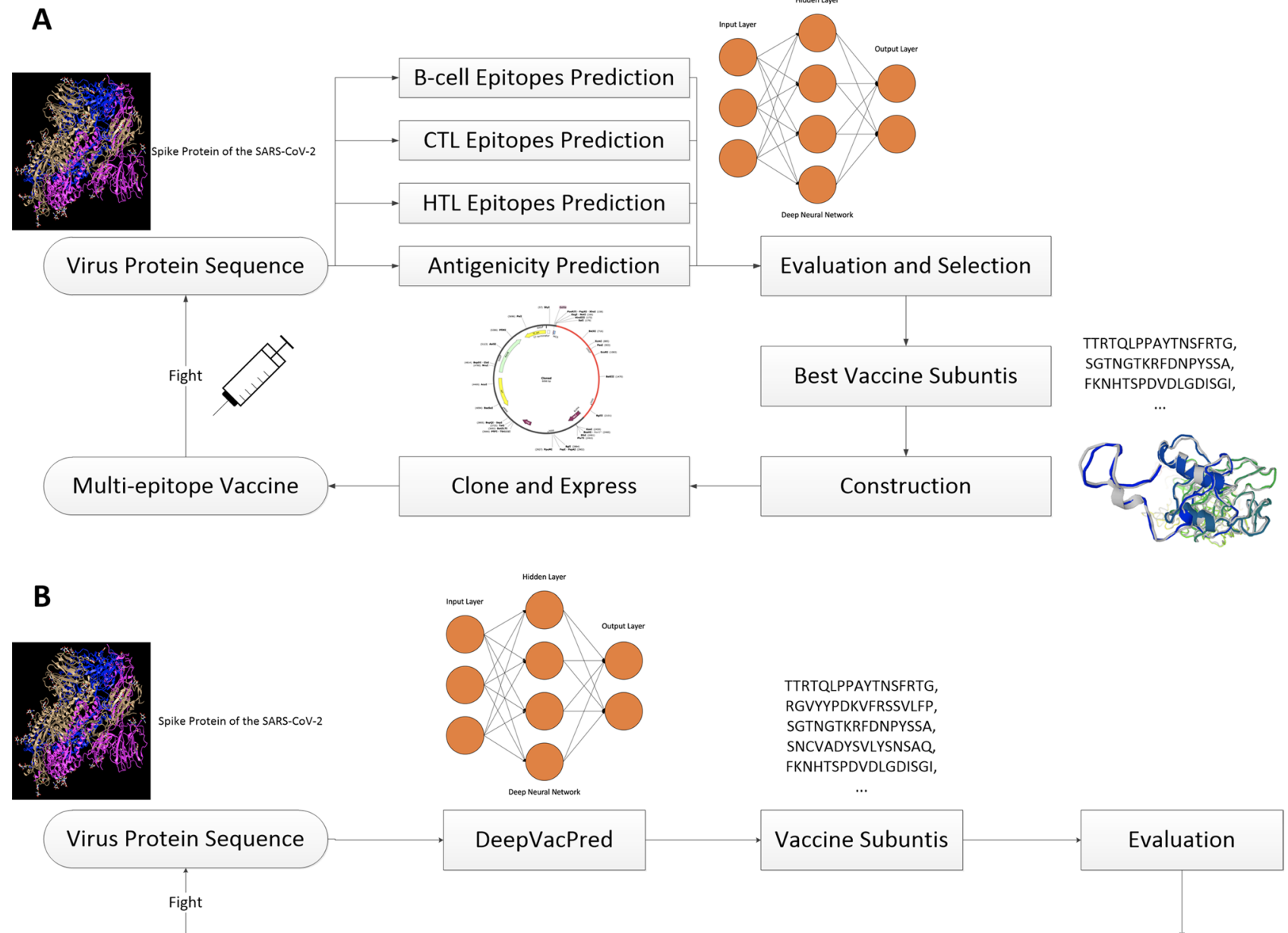

TTRTQLPPAYTNSFRTG, RGVYYPDKVFRSSVLFP, SGTNGTKRFDNPYSSA SNCVADYSVLYSNSAQ, FKNHTSPDVDLGDISGI,

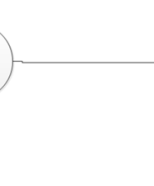

\section{Multi-epitope Vaccine}
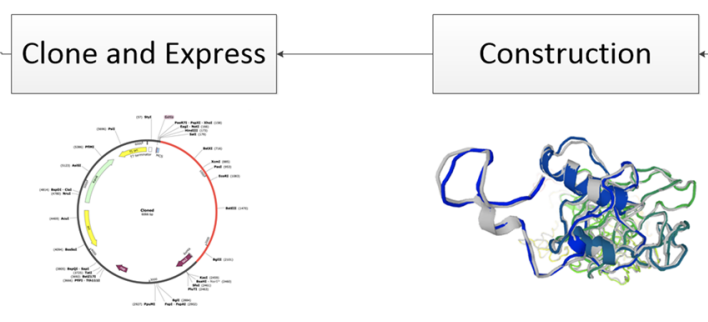

Best Vaccine Subunits

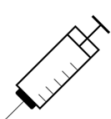

TTRTQLPPAYTNSFRTG SGTNGTKRFDNPYSSA, FKNHTSPDVDLGDISGI,

Figure 1. Schematic Diagram of In Silico Vaccine Design Process. (A) Traditional in silico vaccine design process. We have to use numerous vaccine design tools. The evaluation and subunits selection is very time consuming. No current tool is able to include all the predictions to comprehensively analyze and select out the best vaccine subunits directly. (B) In silico vaccine design by DeepVacPred framework. By replacing the many predictions, evaluations and selections with a DNN architecture inside the DeepVacPred framework, we are able to directly predict a very small number of potential vaccine subunits within a second and start the following evaluation and vaccine construction on a much smaller amount of data.

spike protein of the SARS-CoV-2 can latch onto cells and force the virus through the cell membrane, which enables the virus entry. Previous studies reveal that the spike protein of the SARS-CoV-2 plays a decisive role during the infection. Proteolytic activation of spike protein by host cell proteases is also a critical determinant ${ }^{21}$. It is promising to combat the COVID-19 by inducing the B-cells and T-cells that can perform immune responses against the SARS-CoV-2 spike protein. Hence, in this study, we choose the spike protein sequence of the SARS$\mathrm{CoV}-2$ as the main subject to design our multi-epitope vaccine.

Although the in silico vaccine design approaches are looked at as fairly efficient, they may not be sufficiently fast to keep pace with the emergence of various pandemics. Figure 1A shows the schematic diagram of a traditional in silico vaccine design process. Researchers usually use numerous in silico tools to predict the B-cell, CTL and HTL epitopes on the whole virus proteins ${ }^{22,23}$. The antigenicity and other physicochemical properties of the overlapping fragments are also necessary to be evaluated ${ }^{24}$. To select the best virus protein regions for constructing an efficacious vaccine, we need to carefully and comprehensively evaluate all the predicted results, which creates a large overhead and can be very time consuming. Currently, each in silico vaccine design tool can only achieve one single prediction goal. For example, BepiPred ${ }^{25}$ is a very popular B-cell epitope prediction tool and many researchers use this tool to predict the B-cell epitopes. However, BepiPred can only be used to address 
the one step of B-cell epitope prediction, and when it comes to T-cell epitope prediction, a different tool such as NetMHCpan ${ }^{26}$ is needed. No current tool is able to conduct multiple predictions and comprehensively analyze the results for us at once to directly identify the best vaccine subunits for further construction and evaluation.

To overcome the above challenges of the in silico vaccine design, we propose DeepVacPred, a novel AI-based in silico multi-epitope vaccine design framework. We successfully replace the multiple necessary predictions and the comprehensive evaluations with a deep neural network (DNN) architecture. When the DNN takes one peptide sequence as input, it can then judge whether this input sequence can be a potential vaccine subunit. In the DeepVacPred framework, the number of potential vaccine subunits can be firstly reduced to around 30, then further evaluation and vaccine construction is done on the predicted subunits by reliable and popular in silico methods to construct the final vaccine. Our novel approach aims to achieve a much better efficiency of the in silico vaccine design.

With DeepVacPred, this study designs a multi-epitope vaccine in a novel in silico fashion. We first use the DNN architecture to lock down 26 fragments in the SARS-CoV-2 spike protein as vaccine subunit candidates. Next, we predict the linear B-cell epitopes, CTL epitopes and HTL epitopes to select and construct our final vaccine. We further analyze the human population coverage, antigenicity, allergenicity, toxicity and other physicochemical properties to validate the quality. We also predict the secondary structure and $3 \mathrm{D}$ structure model. This model is eventually refined and validated. Finally, the codon optimization and in silico cloning are performed to check the vaccine genome and protein constructions and ensure its effective expression. In addition, DeepVacPred allows us to quickly check for newly emerging threats caused by the RNA mutations of the SARS-CoV-2. We prove that our vaccine can tackle the virus RNA mutations.

\section{DeepVacPred}

Background. An in silico vaccine design process can be seen as selecting good fragments of the virus proteins, then constructing them together into a final vaccine ${ }^{24}$. A fragment with multiple merits can be selected as a subunit of the final vaccine. For example, an ideal subunit should contain multiple B-cell epitopes and T-cell epitopes and it should have high antigenicity to trigger human protective reactions ${ }^{22,23}$. These merits can be predicted by in silico approaches and currently there are numerous in silico vaccine design tools. However, these tools are designed to address only one of the several predictions at a time. Consequently, researchers have to overcome the time-consuming tasks of analyzing each individual prediction result from different tools while adopting a comprehensive view of the vaccine design. No current tool can take all the necessary merits into consideration and directly predict the vaccine subunit candidates from the virus proteins.

There are two drawbacks to the current situation: (i) We usually need only the best 10-20 subunits to construct the final vaccine while each prediction tool may provide us with hundreds or even thousands of potential locations to choose, which creates a large overhead to comprehensively select out the subunits we need and no current tool can achieve both the prediction and the selection for us. (ii) Nearly $90 \%$ prediction results are eventually discarded because they have only part of the merits, resulting in too much of unnecessary analysis and wasting many computing resources. Consequently, traditional approaches may produce vaccines that are too late or ineffective for pandemics.

In order to improve the efficiency and reliability of the vaccine design process, we improve over state-of-theart tools by providing a DNN approach, DeepVacPred, an efficient in silico vaccine design process to address the afore-mentioned concerns. DeepVacPred directly predicts the best vaccine subunit candidates (the number is within 30) from the virus protein sequences within a second by replacing the prediction and selection with deep neural network architecture, hence promising much higher efficiencies for the vaccine design and test process.

Data collection and dataset design. Reliable data is essential for the performance of supervised learning ${ }^{27}$, thus, it plays a crucial role in the outcome of the vaccine design process. We collected 5000 latest known B-cell epitopes (B) and 2000 known T-cell epitopes containing both MHC (major histocompatibility complex)-1 and MHC-2 binders ${ }^{28}$ (T) from the IEDB database, combining with the same number of proteins which are not T-cell or B-cell epitopes, forming a dataset of epitopes and non-epitopes. 100 known latest viral protective antigens are selected from the IEDB database, and the same number of proteins without protective functions are randomly selected, combining with the 400 antigens from previous work ${ }^{29}$, forming a dataset with 600 antigens.

DeepVacPred is built based on supervised learning on a subtly designed dataset. To directly predict the vaccine subunit candidates, the protein sequences in the positive dataset must contain at least one T-cell epitope and one B-cell epitope and must be protective antigens. Cartesian Product ${ }^{30}$ is the set that contains all ordered pairs from two sets. Thus, the two Cartesian Products, $\mathrm{T} \times \mathrm{B}$ and $\mathrm{B} \times \mathrm{T}$, which are formed between the collected $\mathrm{B}$-cell epitopes dataset and the T-cell epitopes dataset can cover all the possible combinations of the known B-cell and T-cell epitopes. We use the 600 antigens to train a neural network that can identify protective antigens. We use this neural network on the Cartesian Product to sieve out 706,970 peptides sequences that are predicted to be protective antigens. Those 706,970 peptides contain both B-cell epitopes and T-cell epitopes and are protective antigens, referred in this paper as the positive vaccine dataset. The same number of peptides randomly bridged by negative T-cell and B-cell epitopes form our negative vaccine dataset. The dataset we design addresses the three most important predictions, the B-cell epitopes, T-cell epitopes and antigenicity in the vaccine design process.

All the datasets we collected, designed and created for the DNNs training can be found in the Data Availability section. The descriptions of each dataset are shown in Table 1.

Network training. A multi-layer convolutional neural network (CNN) and a four-layer linear neural network connect together, forming a deep neural network (DNN) with a two-class output. The positive and nega- 


\begin{tabular}{|c|c|c|}
\hline Datasets & Number of peptides & Descriptions \\
\hline $\mathrm{T}$ & 2000 & $\begin{array}{l}\text { Known T-cell epitopes with both MHC-1 and MHC-2 } \\
\text { binders collected from the IEDB database. Used for creat- } \\
\text { ing the vaccine datasets }\end{array}$ \\
\hline B & 5000 & $\begin{array}{l}\text { Known B-cell epitopes collected from the IEDB database. } \\
\text { Used for creating the vaccine datasets }\end{array}$ \\
\hline Protective antigens & 300 & $\begin{array}{l}\text { Known viral protective antigens collected from both the } \\
\text { IEDB database and previous work. Used for training a } \\
\text { DNN to identify protective antigens in order to sieve out } \\
\text { the positive vaccine dataset from the Cartesian Products }\end{array}$ \\
\hline Cartesian products & $2000 \times 5000 \times 2$ & $\begin{array}{l}\text { The Cartesian Products of TxB and BxT. The products } \\
\text { include all the peptides generated from the T and B datasets } \\
\text { which contain at least one T-cell epitope and one B-cell } \\
\text { epitope in each peptide }\end{array}$ \\
\hline NT & 2000 & 2000 peptides which are not T-cell epitopes \\
\hline NB & 5000 & 5000 peptides which are not B-cell epitopes \\
\hline $\mathrm{N}$ protective antigens & 300 & 300 peptides which are not viral protective antigens \\
\hline Positive vaccine dataset & 706,970 & $\begin{array}{l}\text { Sieved out from the Cartesian Products by using the DNN } \\
\text { trained by the protective antigen datasets. Each of the } \\
\text { peptide in this dataset contains at least one T-cell epitope } \\
\text { and one B-cell epitope and the whole sequence is predicted } \\
\text { to be protective antigens. Used for training the DNN to } \\
\text { predict vaccine subunits }\end{array}$ \\
\hline Negative vaccine dataset & 706,970 & $\begin{array}{l}\text { The negative dataset to train the DNN to predict vaccine } \\
\text { subunits. Each peptide in this dataset does not contain at } \\
\text { least one T-cell and one B-cell epitope or it is predicted to } \\
\text { be non-protective antigens }\end{array}$ \\
\hline
\end{tabular}

Table 1. Description of the datasets used for analysis and DNN training.

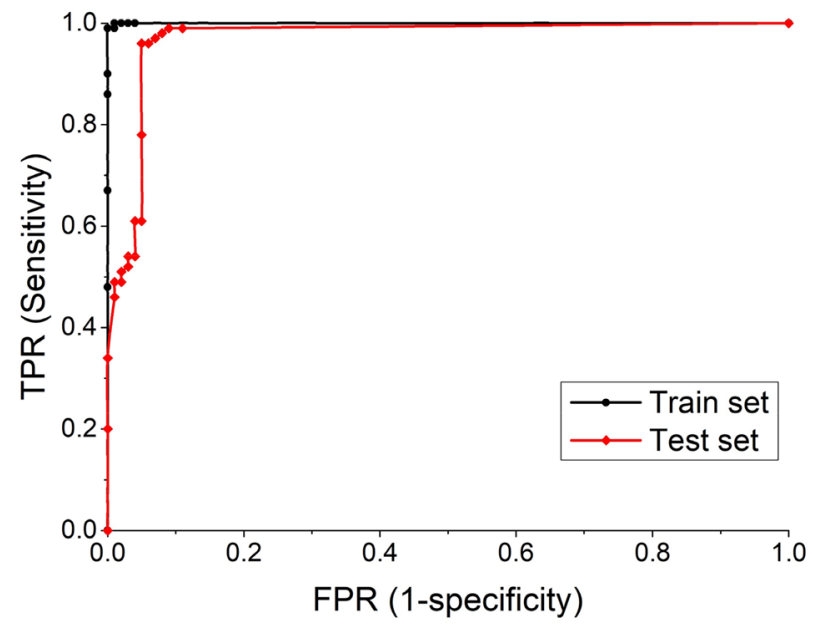

Figure 2. ROC Curves for the DNN-V in DeepVacPred. The area under the ROC curves represent the ability of the DNN-V to classify potential vaccine subunits and non-potential vaccine subunits. The high area under the ROC curves suggests that the DNN-V has strong classification ability and high accuracy at most threshold values.

tive datasets are annotated by Z-descriptors ${ }^{31}$, then converted to the same length of 45 vectors with auto cross covariance (ACC) transformation ${ }^{32}$. Trained by the transformed dataset above, the DNN achieves the classification function to predict whether the input is a protective antigen containing both the B-cell and T-cell epitopes, realizing the ability to directly judge whether a sequence can be a potential vaccine subunit. This DNN is the core part of the rapid vaccine design process of our DeepVacPred framework and we name it as DNN-V. In addition, we train another DNN with the same structure on the T-cell epitope dataset which can judge whether an input sequence can be a T-cell epitope and we name it as DNN-T. The detailed neural network structures, training process and hyper-parameters can be found in "DNN Design and Training in DeepVacPred Framework" in the Methods section.

Validation. ROC curves. Receiver operating characteristic (ROC) curve is a graphical plot that illustrates the diagnostic ability of a binary classifier system as its discrimination threshold is varied ${ }^{33}$. DNN-V is a novel approach that needs to be validated. We use the ROC curves to evaluate the DNN-V in DeepVacPred. We 


\begin{tabular}{|l|l|l|l|l|l|}
\hline Validation & AUC & Threshold & Accuracy & Sensitivity & Specificity \\
\hline Train set & 0.9999 & 0.32 & 0.995 & 0.99 & 0.99 \\
\hline Test set & 0.9703 & 0.5 & 0.95 & 0.95 & 0.95 \\
\hline
\end{tabular}

Table 2. DeepVacPred Validation. For the Training Set, we reach the highest accuracy of 0.995 if the threshold value is set at 0.32 . At this threshold value, the sensitivity and specificity are 0.99 each. For the Testing Set, we reach the highest accuracy of 0.95 if the threshold value is set at 0.5 . At this threshold value, the sensitivity and specificity are 0.95 each. The experimental data shows high accuracy and strong classification ability of the proposed DeepVacPred framework.

\begin{tabular}{|l|l|l|l|l|}
\hline Location & Proteins & Start & End & Number of vaccine subunits \\
\hline Location 1 & Spike & 6 & 36 & 2 \\
\hline Location 2 & Spike & 53 & 104 & 3 \\
\hline Location 3 & Spike & 105 & 167 & 8 \\
\hline Location 4 & Spike & 206 & 322 & 22 \\
\hline Location 5 & Spike & 352 & 585 & 30 \\
\hline Location 6 & Spike & 601 & 741 & 19 \\
\hline Location 7 & Spike & 751 & 862 & 17 \\
\hline Location 8 & Spike & 878 & 981 & 16 \\
\hline Location 9 & Spike & 1034 & 1063 & 1 \\
\hline Location 10 & Spike & 1057 & 1186 & 12 \\
\hline Location 11 & Spike & 1188 & 1218 & 2 \\
\hline
\end{tabular}

Table 3. DeepVacPred first round prediction results. Here we show the number of predicted vaccine subunits for each location.

test the trained DNN-V with two datasets, namely the train set and the test set, each of which containing 200 protein sequences. The training set contains 200 proteins randomly selected from the dataset; we use to train the DNN-V, with 100 positive and 100 negative protein sequences. We also selected known B-cell epitopes and T-cell epitopes that are not in our collected data and use the above steps to form the testing set, also with 100 positive and 100 negative protein sequences. The ROC curves are shown in Fig. 2. The validation data appears in Table 2. The thresholds are ranged from 0 to 1 . The accuracy reported in Table 2 is the greatest value among all thresholds. The sensitivity and specificity values in Table 2 are reported for the case with the highest accuracy. The AUC (Area Under the ROC Curve) value of 0.9703 for the test set which indicates the high accuracy of the classification of DNN-V to identify potential vaccine subunits.

Vaccine design test. The false positive rate (FPR) will fall down to 0 if we set the threshold to a very low value, e.g., 0.0003, since we only care about discarding all the non-candidates. We use the DNN-V in our DeepVacPred framework on the 1273aa spike protein sequence of the SARS-CoV-2. 132 vaccine candidates are predicted. We use BepiPred ${ }^{25}$, NetMHCpan ${ }^{26}$ and Vaxijen ${ }^{34}$ to examine each candidate. All of the candidates contain both T-cell and B-cell epitopes and only 14 of them are predicted by Vaxijen to be non-protective antigens.

DeepVacPred framework. Figure 1B provides the schematic diagram of the vaccine design process using DeepVacPred framework. DeepVacPred first uses DNN-V to predict a very small number of potential vaccine subunits directly from the virus protein sequences. DeepVacPred further uses DNN-T to examine all the overlapping sequences in these subunits and select the subunit candidates which have multiple T-cell epitopes. These two prediction rounds take less than $1 \mathrm{~s}$ and reduce the number of potential vaccine subunits to around 30. Compared to traditional approaches, the most time-consuming subunits selection part can be easily done by DeepVacPred within less than a second, saving a large amount of time and computational resources.

The following steps in the DeepVacPred framework are as follows: (i) selecting the best subunits from only about 30 candidates and (ii) constructing the final vaccine based on the evaluations by various reliable in silico tools, including Linear B-cell epitopes prediction, CTL and HTL epitopes prediction, population coverage analysis, vaccine construction, evaluation of antigenicity, allergenicity, solubility, immunogenicity, toxicity and other physicochemical properties, structure prediction, 3D modeling, in silico cloning, molecular docking and molecular dynamics simulation. Compared to the popular computational process, those evaluations are done on a much smaller amount of data, hence improving the efficiency.

\section{Results}

Data retrieval. The genome sequence of SARS-CoV-2 isolate Wuhan-Hu-1 is retrieved from the NCBI database with accession number MN908947 $7^{35}$. The protein sequences are retrieved according to their translation. Especially, the spike protein (protein ID: QHD43416.1) has a length of 1273 amino acids (aa), and the 


\begin{tabular}{|l|l|l|l|l|}
\hline Vaccine subunits & Protein & Start & End & Peptide sequence \\
\hline Subunit 1 & Spike & 19 & 48 & TTRTQLPPAYTNSFTRGVYYPDKVFRSSVL \\
\hline Subunit 2 & Spike & 34 & 63 & RGVYYPDKVFRSSVLSTQDLFLPFFSNVT \\
\hline Subunit 3 & Spike & 71 & 100 & SGTNGTKRFDNPVLPFNDGVYFASTEKSNI \\
\hline Subunit 4 & Spike & 141 & 170 & LGVYYHKNNKSWMESEFRVYSSANNCTFEY \\
\hline Subunit 5 & Spike & 191 & 220 & FVFKNIDGYFKIYSKHTPINLVRDLPQGFS \\
\hline Subunit 6 & Spike & 209 & 238 & PINLVRDLPQGFSALEPLVDLPIGINITRF \\
\hline Subunit 7 & Spike & 306 & 335 & FTVEKGIYQTSNFRVQPTESIVRFPNITNL \\
\hline Subunit 8 & Spike & 359 & 388 & SNCVADYSVLYNSASFSTFKCYGVSPTKLN \\
\hline Subunit 9 & Spike & 402 & 431 & IRGDEVRQIAPGQTGKIADYNYKLPDDFTG \\
\hline Subunit 10 & Spike & 439 & 468 & NNLDSKVGGNYNYLYRLFRKSNLKPFERDI \\
\hline Subunit 11 & Spike & 480 & 509 & CNGVEGFNCYFPLQSYGFQPTNGVGYQPYR \\
\hline Subunit 12 & Spike & 510 & 539 & VVVLSFELLHAPATVCGPKKSTNLVKNKCV \\
\hline Subunit 13 & Spike & 584 & 613 & ILDITPCSFGGVSVITPGTNTSNQVAVLYQ \\
\hline Subunit 14 & Spike & 626 & 655 & ADQLTPTWRVYSTGSNVFQTRAGCLIGAEH \\
\hline Subunit 15 & Spike & 655 & 684 & HVNNSYECDIPIGAGICASYQTQTNSPRRA \\
\hline Subunit 16 & Spike & 697 & 726 & MSLGAENSVAYSNNSIAIPTNFTISVTTEI \\
\hline Subunit 17 & Spike & 709 & 738 & NNSIAIPTNFTISVTTEILPVSMTKTSVDC \\
\hline Subunit 18 & Spike & 773 & 802 & EQDKNTQEVFAQVKQIYKTPPIKDFGGFNF \\
\hline Subunit 19 & Spike & 805 & 834 & LPDPSKPSKRSFIEDLLFNKVTLADAGFIK \\
\hline Subunit 20 & Spike & 866 & 895 & TDEMIAQYTSALLAGTITSGWTFGAGAALQ \\
\hline Subunit 21 & Spike & 946 & 975 & GKLQDVVNQNAQALNTLVKQLSSNFGAISS \\
\hline Subunit 22 & Spike & 1017 & 1046 & EIRASANLAATKMSECVLGQSKRVDFCGKG \\
\hline Subunit 23 & Spike & 1034 & 1063 & LGQSKRVDFCGKGYHLMSFPQSAPHGVVFL \\
\hline Subunit 24 & Spike & 1094 & 1123 & VFVSNGTHWFVTQRNFYEPQIITTDNTFVS \\
\hline Subunit 25 & Spike & 1156 & 1185 & FKNHTSPDVDLGDISGINASVVNIQKEIDR \\
\hline Subunit 26 & Spike & 1179 & 1208 & IQKEIDRLNEVAKNLNESLIDLQELGKYEQ \\
\hline & & & &
\end{tabular}

Table 4. DeepVacPred second round prediction results. Here we get 26 vaccine subunits for further evaluation and construction. Those 26 vaccine subunits are very likely to have high antigenicity and contain multiple B-cell and T-cell epitopes. With DeepVacPred, those 26 vaccine subunits are reached within less than a second, while it can take days to select those subunits from the virus protein if we use traditional methods. Next, DeepVacPred simply checks the epitopes and other merits on those 26 subunits and constructs the multiepitope vaccine directly from those 26 candidates, which is much more efficient than traditional approaches.

receptor binding domain (RBD) is from 347 to $520 \mathrm{aa}^{20}$. The following experiments are mainly focused on the spike protein region.

DeepVacPred vaccine subunits prediction. All the overlapping protein fragments with a length of $30 \mathrm{aa}$ are generated out of the 1273aa SARs-CoV-2 spike protein sequence. DeepVacPred first tests these 1244 30 aa protein sequences and predicts 132 potential vaccine subunits (see Table 3 ). The DeepVacPred framework further predicts the T-cell epitopes at these locations and discards the subunits which have less than 8 T-cell epitopes $^{36}$. After this prediction, our DeepVacPred provides us with 26 potential vaccine subunits for further evaluation and construction (see Table 4). These subunits are very likely to contain B-cell epitopes and multiple T-cell epitopes. They are also very likely to have high antigenicity and low allergenicity. We start the following in silico vaccine design process directly from the predicted 26 vaccine subunits, which is very efficient.

Linear B-cell epitopes prediction. B-cell epitopes are portions of antigens binding to immunoglobulin or antibody to trigger the B-cells to provide immune response ${ }^{37}$. Linear B-cell epitopes are predicted on the 26 vaccine subunits. Linear B-cell epitopes are predicted by four online servers including BepiPred ${ }^{25}$, SVMtrip ${ }^{38}$, $\mathrm{ABCPred}^{39}$ and $\mathrm{BCPreds}{ }^{40}$. We first use BepiPred for the main prediction and we use the other three servers to check the prediction results by BepiPred. A B-cell epitope predicted by the BepiPred will be discarded if it is not predicted by any of the other three servers. B-cell epitopes must be located in the solvent-exposed region of the antigens to be possible to combine with the $\mathrm{B}$-cell ${ }^{37}$, thus it is essential to predict the surface availability of the structural protein sequence. The surface availability is predicted by Emini tool ${ }^{41,42}$ on the whole SARS-CoV-2 spike protein sequence, and we discarded the epitopes that are not exposed on the surface. After the predictions, we select out 14 vaccine subunits (see Table 5). We further use the RaptorX Property server to evaluate the surface accessibility of the SARS-CoV-2 to validate that the B-cell epitopes in those subunits are well-exposed (see Fig. 3). 


\begin{tabular}{|c|c|c|c|c|c|c|}
\hline Vaccine Subunits & Protein & Start & End & Peptide Sequence & B-cell Epitopes & Emini Score \\
\hline Subunit 1 & Spike & 19 & 48 & $\begin{array}{l}\text { TTRTQLPPAYTNSFTRGVYYPDKV- } \\
\text { FRSSVL }\end{array}$ & TTRTQLPPAYTNSF & 1.937 \\
\hline \multirow{2}{*}{ Subunit 3} & \multirow{2}{*}{ Spike } & \multirow{2}{*}{71} & \multirow{2}{*}{100} & \multirow{2}{*}{$\begin{array}{l}\text { SGTNGTKRFDNPVLPFNDGVY- } \\
\text { FASTEKSNI }\end{array}$} & NGTKRFD & 2.678 \\
\hline & & & & & KSNI & 1.395 \\
\hline Subunit 4 & Spike & 141 & 170 & $\begin{array}{l}\text { LGVYYHKNNKSWMESEFRVYSSAN- } \\
\text { NCTFEY }\end{array}$ & YYHKNNKS & 3.544 \\
\hline Subunit 5 & Spike & 191 & 220 & $\begin{array}{l}\text { FVFKNIDGYFKIYSKHTPIN- } \\
\text { LVRDLPQGFS }\end{array}$ & HTPIN & 1.207 \\
\hline Subunit 9 & Spike & 402 & 431 & $\begin{array}{l}\text { IRGDEVRQIAPGQTGKIADYNYKLP- } \\
\text { DDFTG }\end{array}$ & EVRQIAPGQTGKIADYNYK & 1.775 \\
\hline \multirow{2}{*}{ Subunit 10} & \multirow{2}{*}{ Spike } & \multirow{2}{*}{439} & \multirow{2}{*}{468} & \multirow{2}{*}{$\begin{array}{l}\text { NNLDSKVGGNYNYLYRLFRKSNLKP- } \\
\text { FERDI }\end{array}$} & NNLDSKV & 1.508 \\
\hline & & & & & LFRKSN & 2.403 \\
\hline Subunit 13 & Spike & 584 & 613 & $\begin{array}{l}\text { ILDITPCSFGGVSVITPGTNTSNQ- } \\
\text { VAVLYQ }\end{array}$ & GTNTSN & 1.888 \\
\hline \multirow{2}{*}{ Subunit 15} & \multirow{2}{*}{ Spike } & \multirow{2}{*}{655} & \multirow{2}{*}{684} & \multirow{2}{*}{$\begin{array}{l}\text { HVNNSYECDIPIGAG- } \\
\text { ICASYQTQTNSPRRA }\end{array}$} & HVNNSY & 1.460 \\
\hline & & & & & YQTQTNSPRRAR & 3.849 \\
\hline \multirow{2}{*}{ Subunit 18} & \multirow{2}{*}{ Spike } & \multirow{2}{*}{773} & \multirow{2}{*}{802} & \multirow{2}{*}{$\begin{array}{l}\text { EQDKNTQEVFAQVKQIYKTPPIKD- } \\
\text { FGGFNF }\end{array}$} & QDKNTQ & 4.752 \\
\hline & & & & & KQIYKTPPI & 2.243 \\
\hline Subunit 19 & Spike & 805 & 834 & $\begin{array}{l}\text { LPDPSKPSKRSFIEDLLFNKVTLAD- } \\
\text { AGFIK }\end{array}$ & LPDPSKPSKR & 3.136 \\
\hline \multirow{2}{*}{ Subunit 23} & \multirow{2}{*}{ Spike } & \multirow{2}{*}{1034} & \multirow{2}{*}{1063} & \multirow{2}{*}{$\begin{array}{l}\text { LGQSKRVDFCGKGYHLMSFPQSAPH- } \\
\text { GVVFL }\end{array}$} & GQSKRVDFC & 1.098 \\
\hline & & & & & \begin{tabular}{|l} 
FPQSAPH \\
\end{tabular} & 1.001 \\
\hline Subunit 24 & Spike & 1094 & 1123 & $\begin{array}{l}\text { VFVSNGTHWFVTQRNFYEPQI- } \\
\text { ITTDNTFVS }\end{array}$ & FYEPQIITTD & 1.627 \\
\hline \multirow{2}{*}{ Subunit 25} & \multirow{2}{*}{ Spike } & \multirow{2}{*}{1156} & \multirow{2}{*}{1185} & \multirow{2}{*}{$\begin{array}{l}\text { FKNHTSPDVDLGDISGINASV- } \\
\text { VNIQKEIDR }\end{array}$} & DKYFKNHTSPDVDLGDIS & 1.833 \\
\hline & & & & & IQKEIDR & 1.666 \\
\hline \multirow{2}{*}{ Subunit 26} & \multirow{2}{*}{ Spike } & \multirow{2}{*}{1179} & \multirow{2}{*}{1208} & \multirow{2}{*}{$\begin{array}{l}\text { IQKEIDRLNEVAKNLNESLIDLQEL- } \\
\text { GKYEQ }\end{array}$} & IQKEIDR & 1.666 \\
\hline & & & & & \begin{tabular}{|l|} 
ELGKY \\
\end{tabular} & 2.802 \\
\hline
\end{tabular}

Table 5. Linear B-cell Epitopes Prediction Results. Here, we show the selected 14 vaccine subunits, the contained B-cell epitopes and their Emini scores.
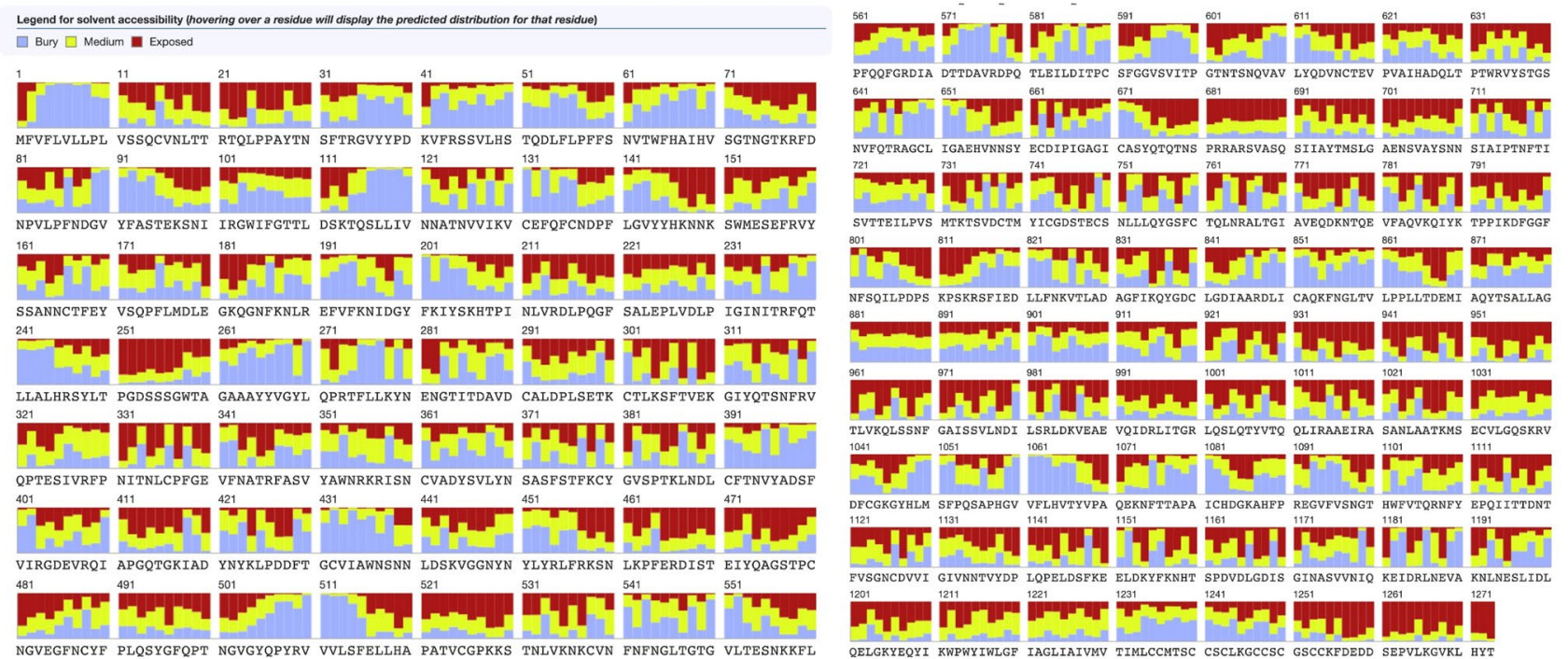

Figure 3. Surface accessibility of the SARS-CoV-2. The red color represents the exposed residues, the yellow color represents the medium exposed residues and the blue color represents the buried residues. In the SARSCoV-2 spike protein, the B-cell epitopes in the 14 vaccine subunits are well-exposed according to the surface accessibility prediction, showing good potential that the B-cell receptor is able to interact with the virus to trigger the immune response.

Cytotoxic T lymphocytes (CTL) epitopes prediction. Cytotoxic T Lymphocytes (CTL) recognize the infected cells by using the MHC class I molecules to bind with certain CTL epitopes ${ }^{26}$. We use NetMHCpan 4.1 server $^{43}$ to predict potential CTL epitopes. All the overlapping 9aa peptide sequences in the 14 vaccine subunits 


\begin{tabular}{|c|c|c|c|c|}
\hline Subunits & Peptide sequence & CTL epitopes & HLA class I alleles and supertypes & HLA score \\
\hline Subunit 1 & TTRTQLPPAYTNSFTRGVYYPDKVFRSSVL & 9 & $\mathrm{~A} 1, \mathrm{~A} 2, \mathrm{~A} 24, \mathrm{~A} 26, \mathrm{~B} 7, \mathrm{~B} 8, \mathrm{~B} 27, \mathrm{~B} 39, \mathrm{~B} 58, \mathrm{~B} 62$ & 4.652 \\
\hline Subunit 3 & $\begin{array}{l}\text { SGTNGTKRFDNPVLPFNDGVYFASTEK- } \\
\text { SNI }\end{array}$ & 6 & $\mathrm{~A} 1, \mathrm{~A} 3, \mathrm{~A} 24, \mathrm{~B} 7, \mathrm{~B} 27, \mathrm{~B} 39, \mathrm{~B} 62$ & 2.492 \\
\hline Subunit 4 & $\begin{array}{l}\text { LGVYYHKNNKSWMESEFRVYSSAN- } \\
\text { NCTFEY }\end{array}$ & 9 & $\mathrm{~A} 1, \mathrm{~A} 3, \mathrm{~A} 24, \mathrm{~A} 26, \mathrm{~B} 39, \mathrm{~B} 40, \mathrm{~B} 58, \mathrm{~B} 62$ & 6.124 \\
\hline Subunit 5 & FVFKNIDGYFKIYSKHTPINLVRDLPQGFS & 9 & $\mathrm{~A} 1, \mathrm{~A} 2, \mathrm{~A} 24, \mathrm{~A} 26, \mathrm{~B} 7, \mathrm{~B} 8, \mathrm{~B} 27, \mathrm{~B} 39, \mathrm{~B} 58, \mathrm{~B} 62$ & 7.131 \\
\hline Subunit 9 & $\begin{array}{l}\text { IRGDEVRQIAPGQTGKIADYNYKLPDD- } \\
\text { FTG }\end{array}$ & 6 & $\mathrm{~A} 2, \mathrm{~A} 3, \mathrm{~B} 7, \mathrm{~B} 27, \mathrm{~B} 62$ & 3.092 \\
\hline Subunit 10 & $\begin{array}{l}\text { NNLDSKVGGNYNYLYRLFRKSNLKP- } \\
\text { FERDI }\end{array}$ & 9 & $\mathrm{~A} 1, \mathrm{~A} 3, \mathrm{~A} 24, \mathrm{~B} 8, \mathrm{~B} 27, \mathrm{~B} 39, \mathrm{~B} 62$ & 4.326 \\
\hline Subunit 13 & ILDITPCSFGGVSVITPGTNTSNQVAVLYQ & 5 & $\mathrm{~A} 1, \mathrm{~A} 3, \mathrm{~A} 24, \mathrm{~B} 8, \mathrm{~B} 27, \mathrm{~B} 39, \mathrm{~B} 62$ & 5.837 \\
\hline Subunit 15 & HVNNSYECDIPIGAGICASYQTQTNSPRRA & 3 & $\mathrm{~A} 1, \mathrm{~B} 7, \mathrm{~B} 40, \mathrm{~B} 62$ & 0.211 \\
\hline Subunit 18 & $\begin{array}{l}\text { EQDKNTQEVFAQVKQIYKTPPIKDFG- } \\
\text { GFNF }\end{array}$ & 7 & $\mathrm{~A} 1, \mathrm{~A} 2, \mathrm{~A} 3, \mathrm{~A} 24, \mathrm{~A} 26, \mathrm{~B} 8, \mathrm{~B} 39, \mathrm{~B} 40, \mathrm{~B} 62$ & 4.282 \\
\hline Subunit 19 & LPDPSKPSKRSFIEDLLFNKVTLADAGFIK & 8 & $\begin{array}{l}\mathrm{A} 1, \mathrm{~A} 2, \mathrm{~A} 3, \mathrm{~A} 24, \mathrm{~B} 7, \mathrm{~B} 8, \mathrm{~B} 27, \mathrm{~B} 39, \mathrm{~B} 40, \\
\mathrm{~B} 58, \mathrm{~B} 62\end{array}$ & 5.763 \\
\hline Subunit 23 & $\begin{array}{l}\text { LGQSKRVDFCGKGYHLMSFPQSAPHGV- } \\
\text { VFL }\end{array}$ & 8 & $\mathrm{~A} 1, \mathrm{~A} 2, \mathrm{~A} 3, \mathrm{~A} 24, \mathrm{~A} 26, \mathrm{~B} 7, \mathrm{~B} 8, \mathrm{~B} 39, \mathrm{~B} 58, \mathrm{~B} 62$ & 6.167 \\
\hline Subunit 24 & $\begin{array}{l}\text { VFVSNGTHWFVTQRNFYEPQIITTDNT- } \\
\text { FVS }\end{array}$ & 8 & $\mathrm{~A} 2, \mathrm{~A} 3, \mathrm{~A} 24, \mathrm{~A} 26, \mathrm{~B} 27, \mathrm{~B} 39, \mathrm{~B} 58, \mathrm{~B} 62$ & 5.66 \\
\hline Subunit 25 & FKNHTSPDVDLGDISGINASVVNIQKEIDR & 4 & $\mathrm{~A} 2, \mathrm{~A} 26, \mathrm{~B} 39$ & 1.341 \\
\hline Subunit 26 & IQKEIDRLNEVAKNLNESLIDLQELGKYEQ & 5 & $\mathrm{~A} 1, \mathrm{~A} 2, \mathrm{~B} 7, \mathrm{~B} 8, \mathrm{~B} 40, \mathrm{~B} 62$ & 3.26 \\
\hline
\end{tabular}

Table 6. CTL epitopes prediction results.

\begin{tabular}{|l|l|l|l|c|}
\hline Subunits & Peptide sequence & HTL epitopes & HLA class II (HLA-DRB1 $* 01)$ alleles & HLA score \\
\hline Subunit 1 & TTRTQLPPAYTNSFTRGVYYPDKVFRSSVL & 9 & $01,03,04,07,08,09,10,11,13,15,16$ & 18.031 \\
\hline Subunit 3 & $\begin{array}{l}\text { SGTNGTKRFDNPVLPFNDGVYFASTEK- } \\
\text { SNI }\end{array}$ & 10 & $01,04,07,08,09,10,12,13,14,15$ & 9.07 \\
\hline Subunit 4 & $\begin{array}{l}\text { LGVYYHKNNKSWMESEFRVYSSAN- } \\
\text { NCTFEY }\end{array}$ & 9 & $04,08,10,11,13,15,16$ & 7.38 \\
\hline Subunit 5 & FVFKNIDGYFKIYSKHTPINLVRDLPQGFS & 14 & $01,03,04,07,08,09,10,11,12,13,14,15,16$ & 26.785 \\
\hline Subunit 9 & $\begin{array}{l}\text { IRGDEVRQIAPGQTGKIADYNYKLPDD- } \\
\text { FTG }\end{array}$ & 7 & $01,07,09,10,14$ & 4.932 \\
\hline Subunit 10 & $\begin{array}{l}\text { NNLDSKVGGNYNYLYRLFRKSNLKP- } \\
\text { FERDI }\end{array}$ & 8 & $07,08,11,13,14,16$ & 12.14 \\
\hline Subunit 13 & ILDITPCSFGGVSVITPGTNTSNQVAVLYQ & 2 & 10 & 0.618 \\
\hline Subunit 15 & $\begin{array}{l}\text { HVNNSYECDIPIGAG- } \\
\text { ICASYQTQTNSPRRA }\end{array}$ & 4 & $01,03,04,09,10,16$ & 3.986 \\
\hline Subunit 18 & $\begin{array}{l}\text { EQDKNTQEVFAQVKQIYKTPPIKDFG- } \\
\text { GFNF }\end{array}$ & 9 & $03,04,07,08,09,10,11,12,13,14,15,16$ & 21.858 \\
\hline Subunit 19 & LPDPSKPSRSFIEDLLFNKVTLADAGFIK & 8 & $03,04,08,09,10,11,14$ & 5.479 \\
\hline Subunit 23 & $\begin{array}{l}\text { LGQSKRVDFCGKGYHLMSFPQSAPHGV- } \\
\text { VFL }\end{array}$ & 4 & $01,04,08,10,11$ & 2.996 \\
\hline Subunit 24 & $\begin{array}{l}\text { VFVSNGTHWFVTQRNFYEPQIITTDNT- } \\
\text { FVS }\end{array}$ & 8 & $03,04,07,08,09,10,11,12,13,14,15,16$ & 11.56 \\
\hline Subunit 25 & FKNHTSPDVDLGDISGINASVVNIQKEIDR & 8 & $01,04,07,08,09,10,11,12,13,14,15$ & 11.925 \\
\hline Subunit 26 & IQKEIDRLNEVAKNLNESLIDLQELGKYEQ & 6 & $08,11,12,14$ & 3.489 \\
\hline
\end{tabular}

Table 7. HTL epitopes prediction results.

are tested with the most common 12 human-leukocyte-antigen (HLA) Class I alleles including HLA-A1, HLAA2, HLA-A3, HLA-A24, HLA-A26, HLA-B7, HLA-B8, HLA-B27, HLA-B39, HLA-B44, HLA-B58 and HLA-B62 to evaluate their binding affinities and predict potential CTL epitopes ${ }^{26,44}$. The total HLA score is calculated for each vaccine subunits. The results are shown in Table 6.

Helper T lymphocytes (HTL) epitopes prediction. Helper T Lymphocytes (HTL) help the activity of other immune cells and they recognize the infection by using MHC class II molecules to bind with certain HTL epitopes $^{45}$. We use NetMHCIIpan 4.0 server $^{46}$ to predict potential HTL epitopes. All the overlapping 15aa peptide sequences in the 14 vaccine subunits are tested with the most common 13 HLA Class II alleles including HLA-DRB1-0101, HLA-DRB1-0301, HLA-DRB1-0401, HLA-DRB1-0701, HLA-DRB1-0801, HLA-DRB1-0901, HLA-DRB1-1001, HLA-DRB1-1101, HLA-DRB1-1201, HLA-DRB1-1301, HLA-DRB1-1401, HLA-DRB1-1501, 


\begin{tabular}{|l|l|l|l|l|l|}
\hline Vaccine subunits & Protein & Start & End & Peptide sequence & Population coverage (worldwide) \% \\
\hline Subunit 1 & Spike & 19 & 48 & TTRTQLPPAYTNSFTRGVYYPDKVFRSSVL & 96.95 \\
\hline Subunit 3 & Spike & 71 & 100 & SGTNGTKRFDNPVLPFNDGVYFASTEKSNI & 83.02 \\
\hline Subunit 4 & Spike & 141 & 170 & LGVYYHKNNKSWMESEFRVYSSANNCTFEY & 81.74 \\
\hline Subunit 5 & Spike & 191 & 220 & FVFKNIDGYFKIYSKHTPINLVRDLPQGFS & 97.04 \\
\hline Subunit 9 & Spike & 402 & 431 & IRGDEVRQIAPGQTGKIADYNYKLPDDFTG & 77.19 \\
\hline Subunit 10 & Spike & 439 & 468 & NNLDSKVGGNYNYLYRLFRKSNLKPFERDI & 78.51 \\
\hline Subunit 13 & Spike & 584 & 613 & ILDITPCSFGGVSVITPGTNTSNQVAVLYQ & 61.44 \\
\hline Subunit 15 & Spike & 655 & 684 & HVNNSYECDIPIGAGICASYQTQTNSPRRA & 68.94 \\
\hline Subunit 18 & Spike & 773 & 802 & EQDKNTQEVFAQVKQIYKTPPIKDFGGFNF & 90.19 \\
\hline Subunit 19 & Spike & 805 & 834 & LPDPSKPSKRSFIEDLLFNKVTLADAGFIK & 76.12 \\
\hline Subunit 23 & Spike & 1034 & 1063 & LGQSKRVDFCGKGYHLMSFPQSAPHGVVFL & 68.38 \\
\hline Subunit 24 & Spike & 1094 & 1123 & VFVSNGTHWFVTQRNFYEPQIITTDNTFVS & 94.90 \\
\hline Subunit 25 & Spike & 1156 & 1185 & FKNHTSPDVDLGDISGINASVVNIQKEIDR & 87.47 \\
\hline Subunit 26 & Spike & 1179 & 1208 & IQKEIDRLNEVAKNLNESLIDLQELGKYEQ & 76.72 \\
\hline
\end{tabular}

Table 8. Worldwide human population coverage analysis results.
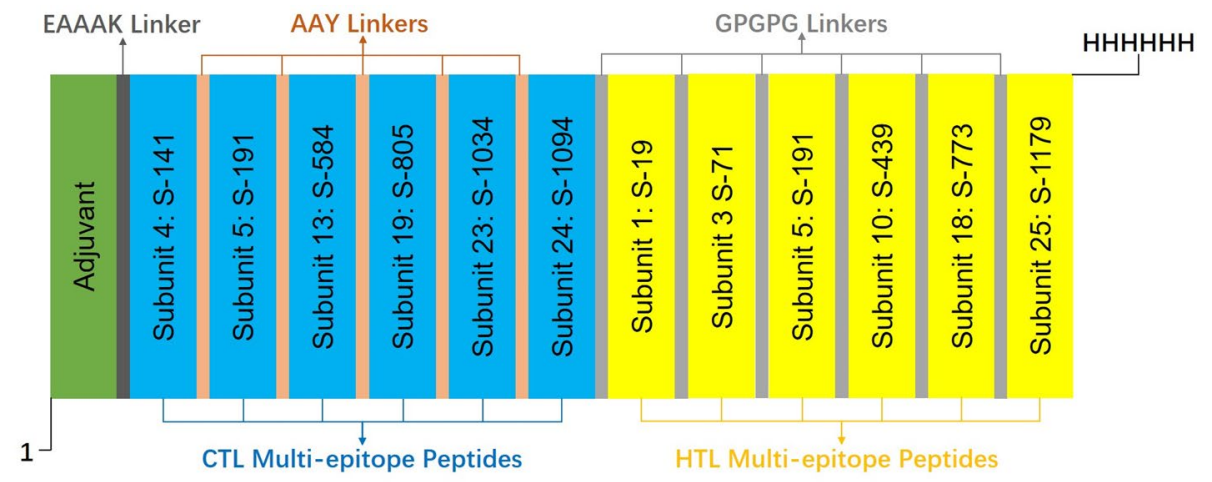

Figure 4. Schematic Presentation of the final Multi-epitope Vaccine. The vaccine is constructed by 11 subunits (Subunit 5 is used twice in both CTL and HTL region for its good performance), an adjuvant and a 6xHis tag, linked by EAAAK, AAY and GPGPG linkers. The final vaccine consists of 694 amino acid residues. It contains 16 B-cell epitopes, 82 CTL epitopes and 89 HTL epitopes.

HLA-DRB1-1601 to evaluate their binding affinities and predict the potential HTL epitopes ${ }^{45,47}$. The total HLA score is calculated for each vaccine subunits. The results appears in Table 7.

Worldwide human population coverage analysis. The vaccine we design should have wide human population coverage. We use the IEDB population coverage analysis tool ${ }^{48}$ to evaluate the worldwide human population coverage of the 14 vaccine subunits. The 25 HLA alleles we used to predict the T-cell epitopes can cover $98.39 \%$ of the human population. The human population coverage of each vaccine subunit is shown in Table 8 . The results suggest that our 14 vaccine subunits can cover a very wide range of human population.

Multi-epitope vaccine construction. We discard Subunits 9, 15 and 26 for their poor performance in the CTL and HTL epitope predictions. We use the remaining 11 vaccine subunits to construct a final multi-epitope vaccine (see Fig. 4). To avoid potential autoimmunity, we perform a BLASTp screening against the Uniprot database on those 11 vaccine subunits. A subunit with a higher-than-35\% identity will be considered as homologous protein with human proteome. Among the 11 vaccine subunits we choose for the final vaccine construction, none of them show high degree of homology with the human proteome. The final vaccine contains an adjuvant, 50 S ribosomal protein L2 ${ }^{49,50}$ (accession no. AXI95322.1), to improve the immune response ${ }^{51}$, linked with the amino $(\mathrm{N})$ terminum of the multi-subunit sequence through an EAAAK linker ${ }^{52}$. The multi-subunit sequence has a CTL multi-epitope peptides region followed by an HTL multi-epitope peptides region. The CTL region is constructed by 6 subunits which have better performance in the CTL epitopes prediction. AAY linkers ${ }^{52}$ are used in this region to fuse the subunits. The HTL region is constructed by 6 subunits which have better performance in the HTL epitopes prediction. GPGPG linkers ${ }^{52}$ are used in this region to fuse the subunits. The two regions are linked through a GPGPG linker. In addition, Subunit 5 is used twice in both CTL and HTL region for its good performance in both CTL and HTL epitope predictions. In the end, a 6xHis tag is added at the C-terminal to 


\begin{tabular}{|l|l|l|l|l|l|l|}
\hline Vaccine subunits & Vaxijen score & Antigen pro score & AllerTOP result & Allergen FP result & $\begin{array}{l}\text { Solubility by } \\
\text { SolPro }\end{array}$ & $\begin{array}{l}\text { Solubility by } \\
\text { protein-sol }\end{array}$ \\
\hline Adjuvant & 0.7447 & 0.8205 & NA & NA & 0.7568 & 0.716 \\
\hline Subunit 1 & 0.2486 & 0.4137 & NA & NA & 0.5890 & 0.684 \\
\hline Subunit 3 & 0.4791 & 0.5923 & NA & NA & 0.8113 & 0.660 \\
\hline Subunit 4 & 0.3891 & 0.7364 & NA & NA & 0.6242 & 0.608 \\
\hline Subunit 5 & 0.4757 & 0.4768 & NA & NA & 0.7819 & 0.686 \\
\hline Subunit 10 & 0.3615 & 0.6256 & NA & NA & 0.6023 & 0.652 \\
\hline Subunit 13 & 0.8318 & 0.4032 & NA & NA & 0.9114 & 0.730 \\
\hline Subunit 18 & 0.2449 & 0.3076 & NA & NA & 0.9928 & 0.742 \\
\hline Subunit 19 & 0.3605 & 0.4991 & NA & NA & 0.7831 & 0.636 \\
\hline Subunit 23 & 0.6713 & 0.7355 & NA & NA & 0.6891 & 0.640 \\
\hline Subunit 24 & 0.4012 & 0.5211 & NA & NA & 0.9747 & 0.545 \\
\hline Subunit 25 & 0.6035 & 0.7433 & NA & NA & 0.6425 & 0.947 \\
\hline Final Vaccine & 0.5705 & 0.8814 & NA & NA & 0.7555 & 0.723 \\
\hline
\end{tabular}

Table 9. Antigenicity, allergenicity and solubility Evaluation Results. NA: non-allergen. Higher Vaxijen and Antigen Pro scores suggest higher antigenicity. Higher SolPro and Protein-sol scores suggest higher solubility.

\begin{tabular}{|l|l|l|l|l|l|l|l|l|l|}
\hline & Toxicity & Hydropathicity & Charge & $\begin{array}{l}\text { Half-life (in } \\
\text { vitro) }\end{array}$ & $\begin{array}{l}\text { Half-life (in } \\
\text { vivo) }\end{array}$ & $\begin{array}{l}\text { Instability } \\
\text { index }\end{array}$ & Stability & pI \\
weight
\end{tabular}

Table 10. Toxicity and physicochemical properties prediction results. NT: none-toxicity. We use the protein screening mode in the ToxinPred server to check the overlapping peptides in the final vaccine and adjuvant sequence and they do not contain any toxic peptide. For the rest subunits, we directly use the SVM based prediction to predict their toxicity.

help purify and identify the protein ${ }^{53}$. The final vaccine consists of 694 amino acid residues. It contains 16 B-cell epitopes, 82 CTL epitopes and 89 HTL epitopes.

Antigenicity, allergenicity and solubility evaluation. The antigenicity of the final multi-epitope vaccine sequence is evaluated by the Vaxijen 2.0 online server ${ }^{34,54}$ and the AntigenPro server ${ }^{55}$. We also evaluate the antigenicity of each vaccine subunit, including the adjuvant (see Table 9). The Vaxijen score for the whole final vaccine is 0.5705 with a virus model at a threshold of 0.4 , suggesting a high antigenicity of our final vaccine. The AllergenFP 1.0 server and AllerTOP 2.0 server ${ }^{56}$ predict the final vaccine and its every subunit to be non-allergenic (see Table 9). The solubility of the final vaccine and its every subunit is evaluated by SolPro ${ }^{57}$ and Protein-sol server ${ }^{58}$. The predicted values suggest that our final vaccine and its every subunit have good solubility (see Table 9).

Toxicity and physicochemical properties analysis. The vaccine must not have toxicity potential and the physicochemical properties are also important to evaluate how the vaccine interacts with the environments ${ }^{59}$. We use the ToxinPred server ${ }^{60}$ to predict the toxicity. Other physicochemical properties, including hydropathicity, charge, half-life, instability index, pI (theoretical isoelectric point value) and molecule wheight, are predicted by ExPASy ProtParam Tool ${ }^{61}$. For the whole final vaccine sequence and the adjuvant sequence, we use the pro- 
\begin{tabular}{lllllllllllll|l|l|l|l|l|l|l|l|l|l|l|l|l|l|l|l|l|l|l|l|l|l|l|l|l|l|l|l|l|l|l|l|l|l|l|l|l|l|l|l|l}
1 & M & A & I & I & K & $\mathbf{Y}$ & $\mathbf{K}$ & $\mathbf{P}$ & $\mathbf{T}$ & $\mathbf{S}$ & $\mathbf{N}$ & $\mathbf{G}$ & $\mathbf{R}$ & $\mathbf{R}$ & $\mathbf{N}$ & $\mathbf{M}$ & $\mathbf{S}$ & $\mathbf{G}$ & $\mathbf{S}$ & $\mathbf{D}$ & $\mathbf{F}$ & $\mathbf{A}$ & $\mathbf{E}$ & $\mathbf{I}$ & $\mathbf{T}$ & $\mathbf{K}$ & $\mathbf{T}$ & $\mathbf{K}$ & $\mathbf{P}$ & $\mathbf{E}$ & $\mathbf{K}$ & $\mathbf{T}$ & $\mathbf{L}$ & $\mathbf{L}$ & $\mathbf{V}$ & $\mathbf{S}$ & $\mathbf{Q}$ & $\mathbf{S}$ & $\mathbf{H}$ & $\mathbf{T}$ & $\mathbf{A}$ & $\mathbf{G}$ & $\mathbf{R}$ & $\mathbf{N}$ & $\mathbf{A}$ & $\mathbf{H}$ & $\mathbf{G}$ & $\mathbf{H}$ & $\mathbf{I}$ & $\mathbf{T}$ & 50
\end{tabular}

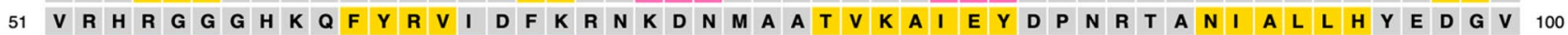
\begin{tabular}{lll|l|l|l|l|l|l|l|l|l|l|l|l|l|l|l|l|l|l|l|l|l|l|l|l|l|l|l|l|l|l|l|l|l|l|l|l|l|l|l|l|l|l|l|l|l|l|l|l|l}
101 & K & S & Y & I & L & A & $\mathbf{P}$ & K & G & L & K & V & G & D & K & V & Y & S & G & D & D & V & D & I & K & V & G & N & T & L & K & L & K & N & I & P & A & G & T & T & I & H & N & I & E & L & K & P & G & K & 150
\end{tabular}

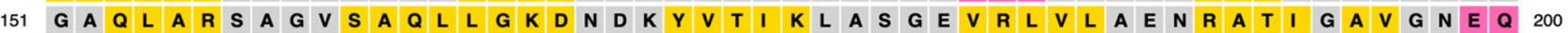

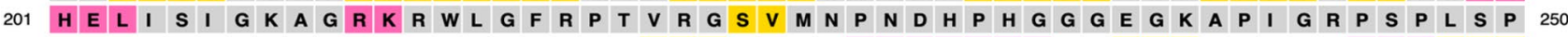

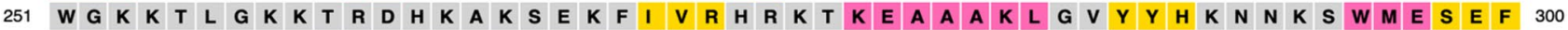

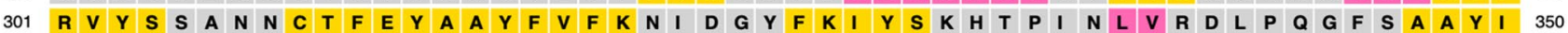

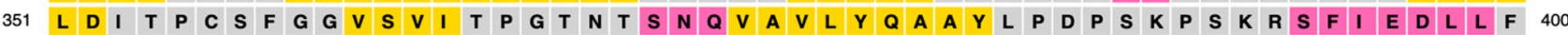

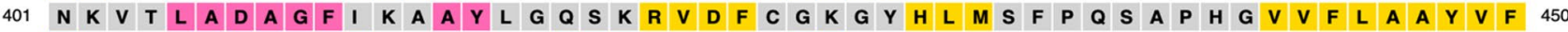

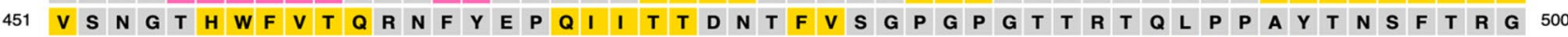

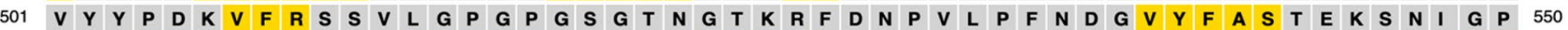

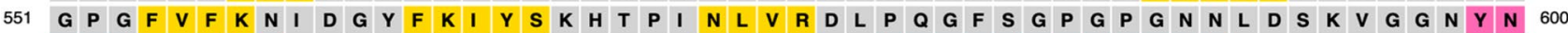

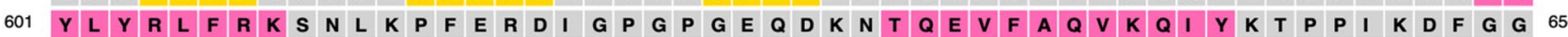

\begin{tabular}{|c|c|c|c|}
\hline Strand & Helix & Coil & Disordered \\
\hline Disordered, protein binding & Putative Domain Boundary & Membrane Interaction & Transmembrane Helix \\
\hline Extracellular & Re-entrant Helix & Cytoplasmic & Signal Peptide \\
\hline
\end{tabular}

Figure 5. Graphical Representation of the Secondary Structure Features. The alpha helix residues are in pink, the beta strand residues are in yellow and the coil residues are in grey. The predicted secondary stucture indicates that the final vaccine constitutes $10.8 \%$ alpha helix, $24.6 \%$ beta strand, and $64.6 \%$ coil, respectively.
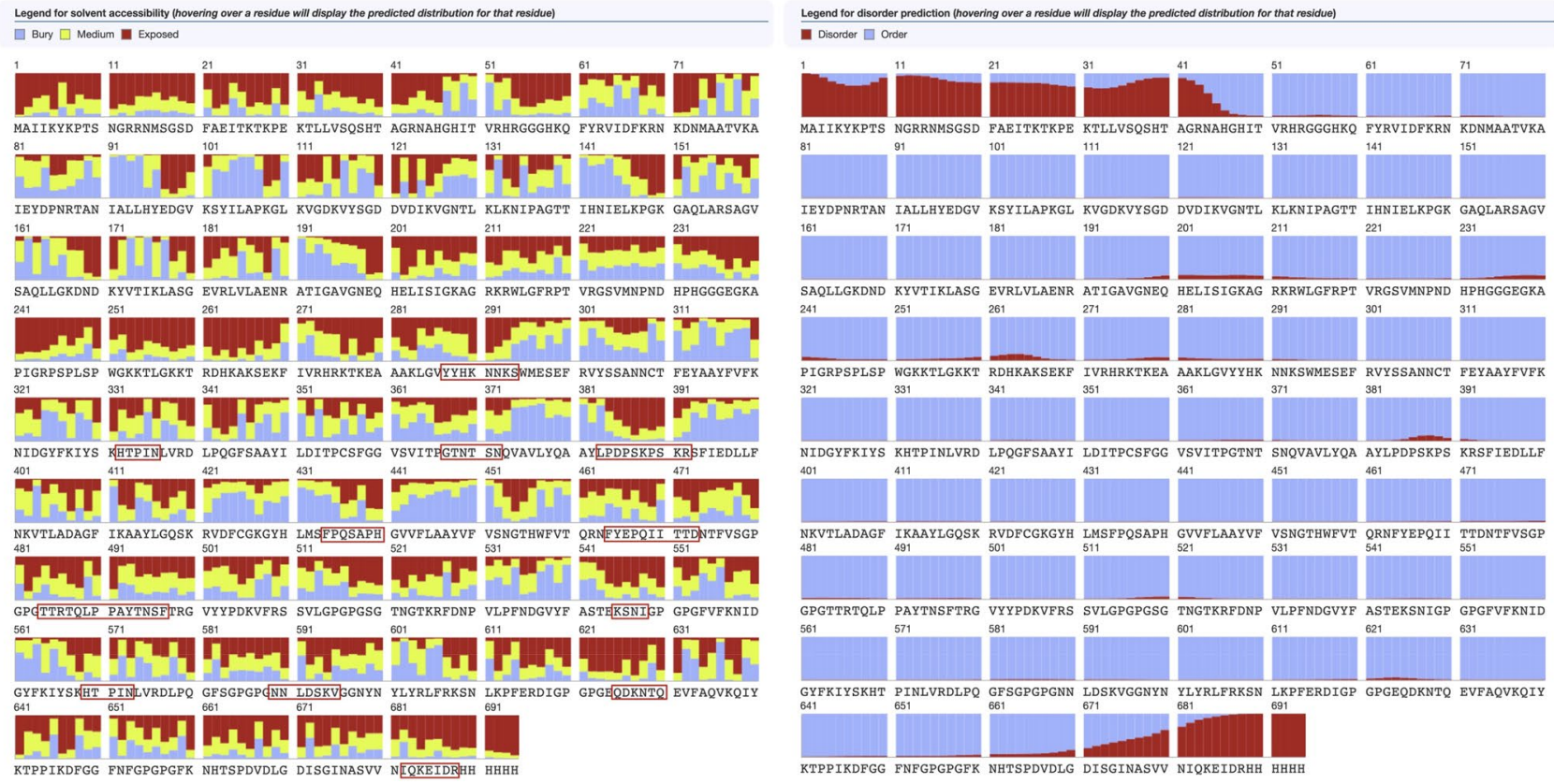

Figure 6. Solvent Accessibility and Disorder Regions Prediction Results. In the solvent accessibility prediction results, the red color represents the exposed residues, the yellow color represents the medium exposed residues and the blue color represents the buried residues. The peptides marked in red boxes are B-cell epitopes. The prediction results show that the B-cell epitopes in the final vaccine have good surface accessibility and also they are not close to each other. In the disorder regions prediction results, the ordered regions are in blue while the disordered regions are in red. A total of 60 residues (8\%) are in disordered regions, showing good order in structure.

tein screening mode in the ToxinPred server to check all its overlapping peptides with length no more than 50 aa. The whole vaccine and the adjuvant do not contain any toxic part peptide. Other subunits and the $6 \mathrm{xHis}$ tag are checked by the SVM prediction mode in the ToxinPred server and all the subunits and the 6xHis tag are nontoxicity. The hydropathicity value of the final vaccine is predicted to be -0.521 . This negative value suggests that our final vaccine is hydrophilic in nature and can interact with water molecules easily ${ }^{62}$. The charge is 37.00 ; this value will decrease in alkaline environment so usually it is better if the charge values are positive. The half-life of the final vaccine is predicted to be $30 \mathrm{~h}$ in vitro and $>20 \mathrm{~h}$ in vivo. An Instability Index of 34.01 is predicted; this 


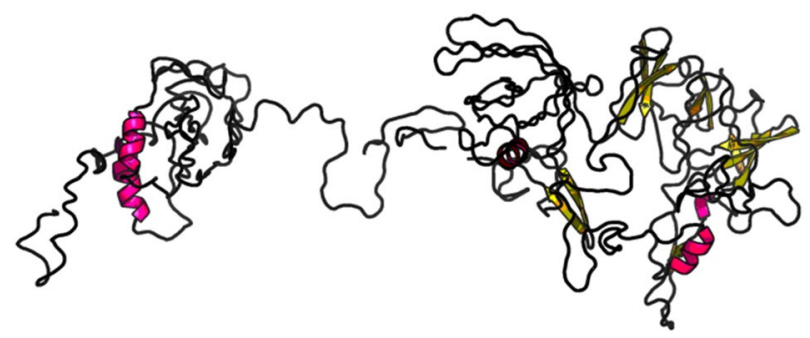

Figure 7. Vaccine 3D Structure Modeling by RaptorX based on the template with PDB ID 3j3vC. All the 694 amino acids in the final vaccine are modeled. The P-value of this model is $4.13 \times 10-14$ and this very low value indicates high quality of this 3D model. The unnormalized Global Distance Test (uGDT) score of this model is 506 (>50), indicating good absolute model quality.

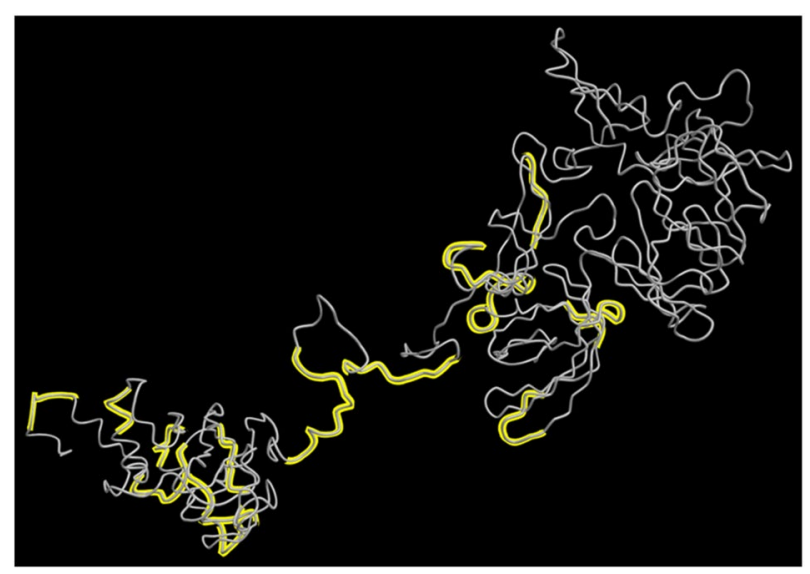

Figure 8. Refined Vaccine 3D Structure Model by GalaxyRefine. This model has a Global Distance Test-High Accuracy (GDT-HA) score of 0.900, a Root Mean Square Deviation (RMSD) score of 0.580, a MolProbity score of 2.618, a clash score of 33.5 and a Ramachandran plot score of $87.5 \%$, showing great overall model quality. The B-cell epitopes in this final vaccine 3D model are highlighted in yellow.

being less than 40 threshold value suggests that our final vaccine is stable. The $\mathrm{pI}$ of the final vaccine is calculated to be 9.75 , which is an alkaline value, indicating its highly basic existence in nature. The molecular weight of the final vaccine is calculated to be $76 \mathrm{kDa}$. We also check the toxocity and physicochemical properties of every subunit and the results are shown in Table 10.

Secondary structure prediction. We use PSIPRED ${ }^{63}$ to generate the secondary structure of our final vaccine. Graphical representation of the secondary structure features are shown in Fig. 5. The predicted secondary stucture indicates that the final vaccine constitutes $10.8 \%$ alpha helix, $24.6 \%$ beta strand, and $64.6 \%$ coil. The solvent accessibility (ACC), and disorder regions (DISO) are predicted by RaptorX Property server ${ }^{64,65}$ (see Fig. 6). Among the 694 amino acid residues in our final vaccine, $44 \%$ are predicted to be exposed, $27 \%$ medium exposed, and $27 \%$ are predicted to be buried. The peptides marked in red boxes in Fig. 6 are the B-cell epitopes, showing good surface accessibility and they are not close to each other. A total of 60 residues (8\%) are predicted to be located in disordered regions.

Vaccine 3D structure modeling. We use the RaptorX server ${ }^{66}$ to build the 3D structure models of our final vaccine. The protein structure with $\mathrm{PDB}$ ID $3 \mathrm{j} 3 \mathrm{vC}$ is predicted by RaptorX to be the best template, based on which this server constructs the 3D structure model of our final vaccine (see Fig. 7). In this model, 100\% (694) amino acids in the final vaccine are modeled in four domains. The P-value quantifies the likelihood of the predicted model being worse than other models generated randomly. The P-value for this model is calculated to be $4.13 \times 10^{-14}$, which is a very low value, suggesting high quality of this $3 \mathrm{D}$ model. The unnormalized Global Distance Test (uGDT) score measures the absolute model quality. The overall uGDT score is predicted to be 506 and being greater than the 50 threshold value for a protein with more than 100 amino acid residues indicates that the $3 \mathrm{D}$ model of our final vaccine is good for further refinement.

Vaccine 3D structure refinement. We use GalaxyRefine server ${ }^{67}$ to refine the 3D structure model of our final vaccine. Among the 5 refined models predicted by GalaxyRefine, we choose the Model 2 shown in Fig. 8 as 


\begin{tabular}{|l|l|l|l|l|l|}
\hline Model & GDT-HA & RMSD & MolProbity & Clash score & Rama favored \\
\hline Initial model & 1.0000 & 0.000 & 4.229 & 137.8 & 78.3 \\
\hline Model 1 & 0.8941 & 0.588 & 2.703 & 33.4 & 87.5 \\
\hline Model 2 & 0.9000 & 0.580 & 2.618 & 33.5 & 87.5 \\
\hline Model 3 & 0.8922 & 0.590 & 2.657 & 33.9 & 87.2 \\
\hline Model 4 & 0.8966 & 0.583 & 2.698 & 33.7 & 87.3 \\
\hline Model 5 & 0.8977 & 0.582 & 2.632 & 34.0 & 87.5 \\
\hline
\end{tabular}

Table 11. Quality scores of the models predicted by GalaxyRefine.

our final vaccine model based on its model quality scores (see Table 11). The predicted B-cell epitopes are highlighted in yellow, showing good surface accessibility. Global Distance Test-High Accuracy (GDT-HA) score measures the similarity between two protein structures. The GDT-HA score between this refined model and the initial model reaches a high value of 0.900 , indicating that they have high similarity. The distance between atoms is measured by the Root Mean Square Deviation (RMSD) score. Lower RMSD value suggests better stability and usually an RMSD score ranges between 0 and 1.2 is acceptable. This model has an RMSD score of 0.580 . Such RMSD score indicates stable protein structure. Molprobity score reflects the crystallographic resolution of the model. The MolProbity score of our identified vaccine model is 2.618 , which is much lower than the initial model, showing that the refinement has lowered the critical errors of the 3D model. The Clash Score reflects the number of unfavorable all-atom steric overlaps and the refinement reduced the clash score of the model from 137.8 to 33.5, improving the model stability to a high level. The Ramachandran plot score represents the size of energetically favoured regions and usually a value greater than $85 \%$ is acceptable. The Ramachandran plot score has been improved from 78.3 to $87.5 \%$ by the refinement. The quality scores of the refined model shows good overall quality.

Vaccine 3D structure validation. We use ProSA-web ${ }^{68}$ to validate the overall model quality of the refined final vaccine model. ProSA predicts a Z-score of -6.51 (see Fig. 9) for the refined model, which is lying inside the score range of the comparable sized native proteins, indicating good overall model quality. ProSA also checks the local model quality and the residue scores are plotted in Fig. 9. Negative values suggest no erroneous parts of the model structure. We also use RAMPAGE server to do the Ramachandran plot analysis and it reveals a Ramachandran plot score of $87.5 \%$, which is consistent with the results of GalaxyRefine.

Conformational B-cell epitope prediction. The structure and folding of the new protein can result in new conformational B-cell epitopes which requires additional predictions. We use ElliPro server ${ }^{69}$ to predict the conformational B-cell epitopes in the refined 3D model. The ElliPro server predicts 6 new conformational B-cell epitopes which involved 387 residues with scores ranging from 0.531 to 0.963 . The detailed 3D model and information of those 6 epitopes are shown in Fig. 10.

Codon optimization and in silico cloning. We analyze the cloning and expression efficiency and optimize the codon usage of vaccine construct in E. coli (Escherichia coli) strain K12) by Java Codon Adaptation Tool $^{70}$. The length of the optimized codon sequence is 2082 nucleotides. Its Codon Adaptation Index (CAI) is 0.997 , and the average GC content is $50.73 \%$, indicating a great potential of good expression of the final vaccine in the E. coli host. After the optimization, we use the SnapGene tool to insert the codon sequences into pET28a $(+)$ vector for cloning ${ }^{71}$ (see Fig. 11). The codon sequence of the final vaccine is presented in red, which is the 2082 bp gene sequence generated by the JCat server. The pET28a $(+)$ expression vector is in black. The codon sequence is inserted between Eco53KI (188) and EcoRV (1573), forming a clone with a total length of $6066 \mathrm{bp}$.

Molecular docking. Molecular docking can evaluate the interactions between a ligand molecule and the receptor molecule to check the stability and binding affinity of their docked complex. Toll-like receptor 4 is an important human protein for pathogen recognition and immune response. Consequently, we choose TLR4 as the immune receptor to perform the molecular docking. We use the ClusPro 2.0 server $^{72}$ to perform the molecular docking between the refined 3D model of our final vaccine and the TLR4 (PDB ID: 4G8A) immune receptor. Among all the generated docking model, we select the one with the lowest energy score of -1311.5 as the best docked complex, suggesting that the vaccine model occupies the receptor properly and indicating good binding affinity (see Fig. 12).

Molecular dynamics simulation of the vaccine-receptor complex. To evaluate the stability and physical movements of the vaccine-TLR4 docked complex ${ }^{17,73}$, we perform molecular dynamics simulation by the iMOD server ${ }^{74}$. The main-chain deformability is shown in Fig. 13a. The locations with hinges are regions with high deformability. The B-factor values calculated by normal mode analysis are proportional to root mean square (see Fig. 13b). B-factor values quantify the uncertainty of each atom. Figure $13 \mathrm{c}$ presents the eigenvalues which are closely related to the energy required to deform the structure and the eigenvalue of the complex is $5.426 \times 10^{-6}$. The covariance matrix between the pairs of residues is shown in Fig. 13d, indicating their correlations (red: correlated, white: uncorrelated, blue: anti-correlated). The elastic network model is shown in Fig. 13e, 
Overall model quality

Z-Score: -6.51

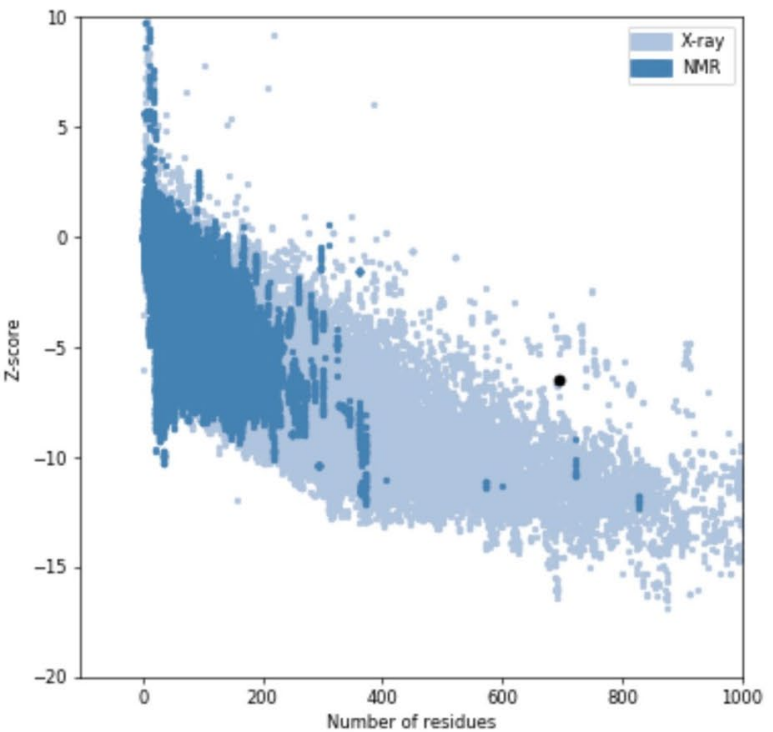

Local model quality

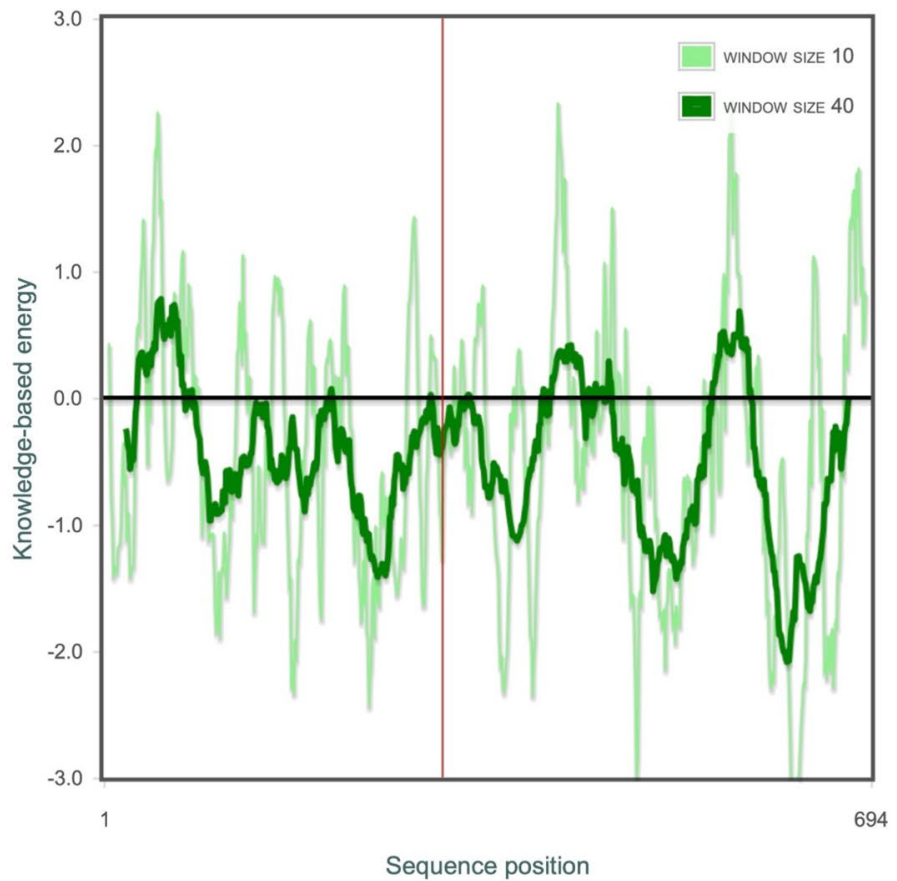

Figure 9. Vaccine 3D Structure Validation by ProSA-web. The Z-score of the refined model is -6.51 which is lying inside the score range. ProSA-web also plots the residues scores to check the local model quality and the negative values suggest no erroneous parts of the model structure.

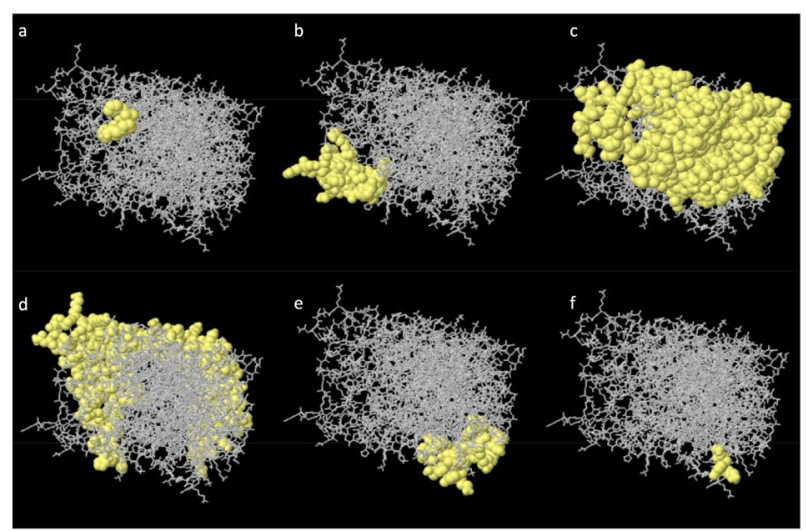

Figure 10. The 3D model of the 6 predicted conformational B-cell epitopes in the refined final vaccine structure. The yellow parts are the conformational B-cell epitopes and the grey parts are the rest of the residues. (a) 3 residues with a score of 0.963 . (b) 30 residues with a score of 0.757 . (c) 167 residues with a score of 0.711 . (d) 161 residues with a score of 0.688. (e) 23 residues with a score of 0.59. (f) 3 residues with a score of 0.531.

suggesting the connection between atoms and springs. The molecular dynamic simulation results suggest that our vaccine model is stable.

RNA mutations. As the SARS-CoV-2 spreads all over the world, its RNA sequence is going through mutations, translating out different virus proteins. Such mutations can have influences on the epitope based vaccines, since a single amino acid difference can change the epitope prediction results. Therefore it is important to prove that the proposed final multi-epitope vaccine can tackle the mutations. With our DeepVacPred, we are also able to quickly examine the mutated protein sequences to search for new potential vaccine subunits.

The RNA sequence we use to translate the spike protein and design the vaccines is from Wuhan, which is the place of the original virus ${ }^{35}$. The RNA mutations lead to three most frequent changes in the spike protein area of the SARS-CoV-2 and each of the changes contains one amino acid change ${ }^{75}$. Table 12 shows the mutation details. 

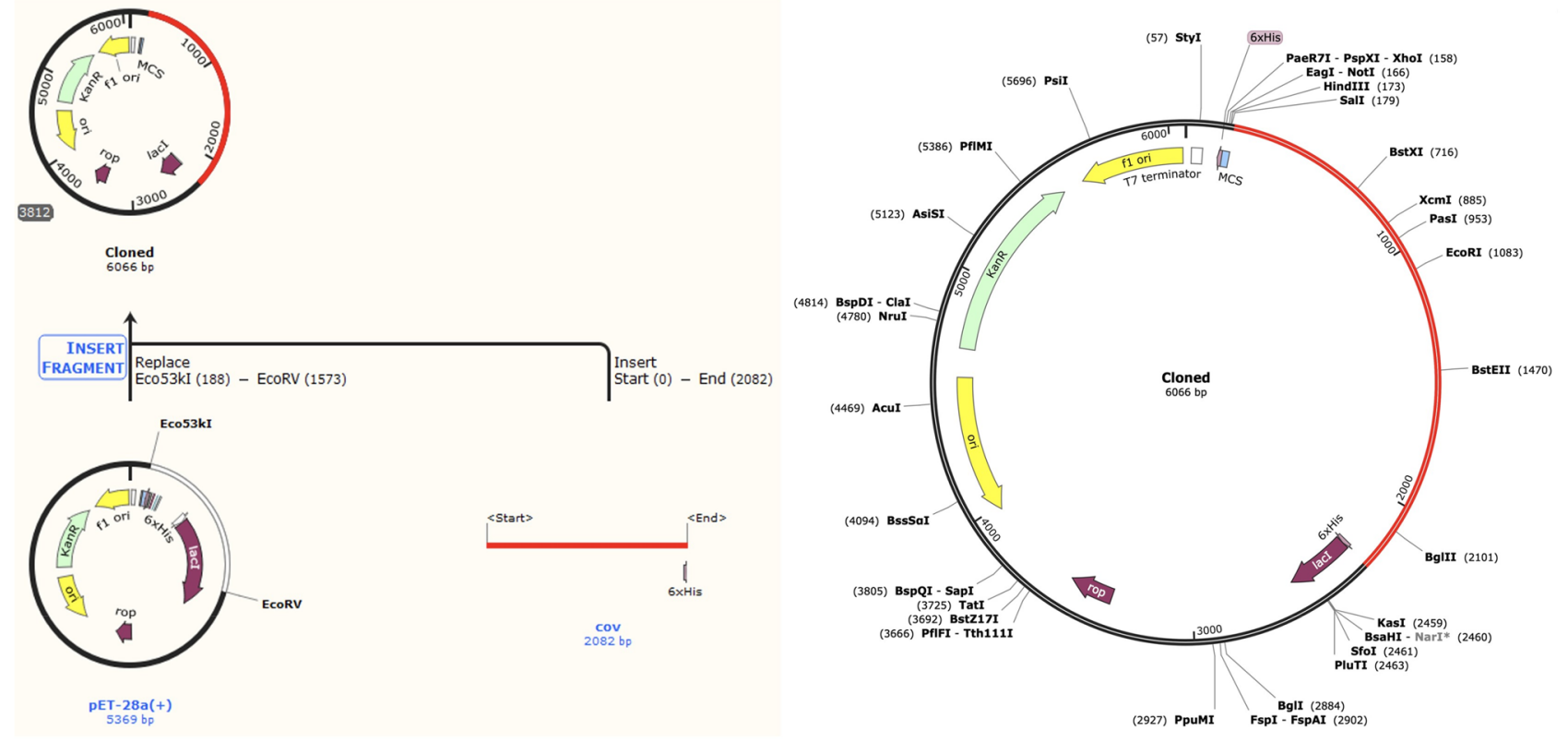

Figure 11. Final Vaccine in silico cloning into the $\mathrm{pET} 28 \mathrm{a}(+)$ vector. The codon sequence of the final vaccine is in red, which is a 2082 bp gene sequence generated by the JCat server. The pET28a(+) expression vector is in black. The codon sequence is inserted between Eco53KI (188) and EcoRV (1573), forming a clone with a toal length of $6066 \mathrm{bp}$. This image was created by SnapGene 5.1 .5 software (from Insightful Science; available at https ://www.snapgene.com).

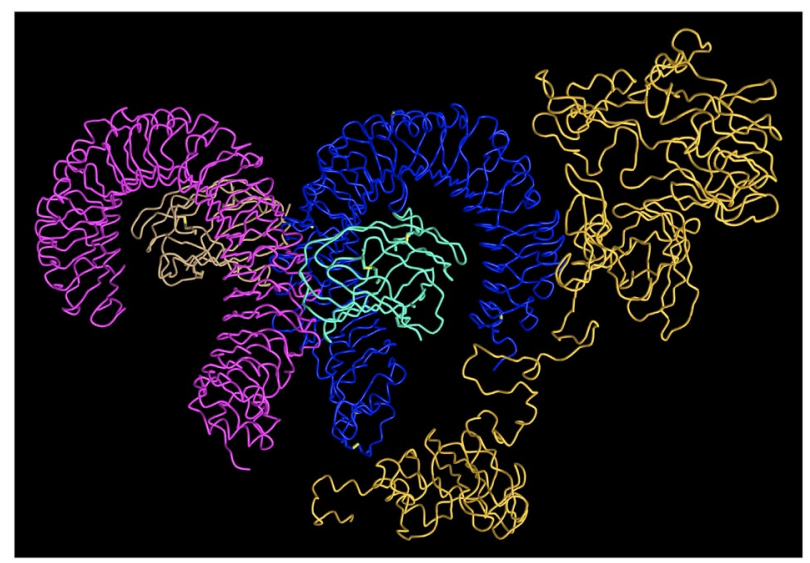

Figure 12. The docked complex of the vaccine model and the TLR4 immune receptor. The vaccine protein is in yellow and the rest of the residues is the TLR4 receptor. The lowest energy score of this complex model is -1311.5 , indiating good binding affinity.

The mutation at the 614 aa in spike protein from $D$ to $G$ is the most frequent mutation with 116 known isolates $^{75}$. This mutation is very common in many cities in North America. In Europe and South America the D614G mutation occurs in less than 10 isolates. This change has no influence on the final multi-epitope vaccine since it does not contain the 614aa of the spike protein. With DeepVacPred, we are also able to quickly check and identify whether the mutation can create new potential vaccine subunits. We input the mutated protein sequence into DeepVacPred and the predicted subunits are the same as the original virus.

At 476 aa in spike protein there is a frequent mutation from $\mathrm{G}$ to $\mathrm{S}$, which occurs in 3 isolates from Washington $\mathrm{DC}^{75}$. This mutation has no influence on the final multi-epitope vaccine since it does not contain the 476aa of the spike protein. We input the mutated protein sequence into DeepVacPred and the predicted subunits are the same as the original virus.

At 483aa in spike protein there is a frequent mutation from $\mathrm{V}$ to $\mathrm{A}$, which occurs in 6 isolates from Washington $\mathrm{DC}^{75}$. This mutation has no influence on the final multi-epitope vaccine since it does not contain the 483aa of the spike protein. We input the mutated protein sequence into DeepVacPred and the predicted subunits are the same as the original virus. 

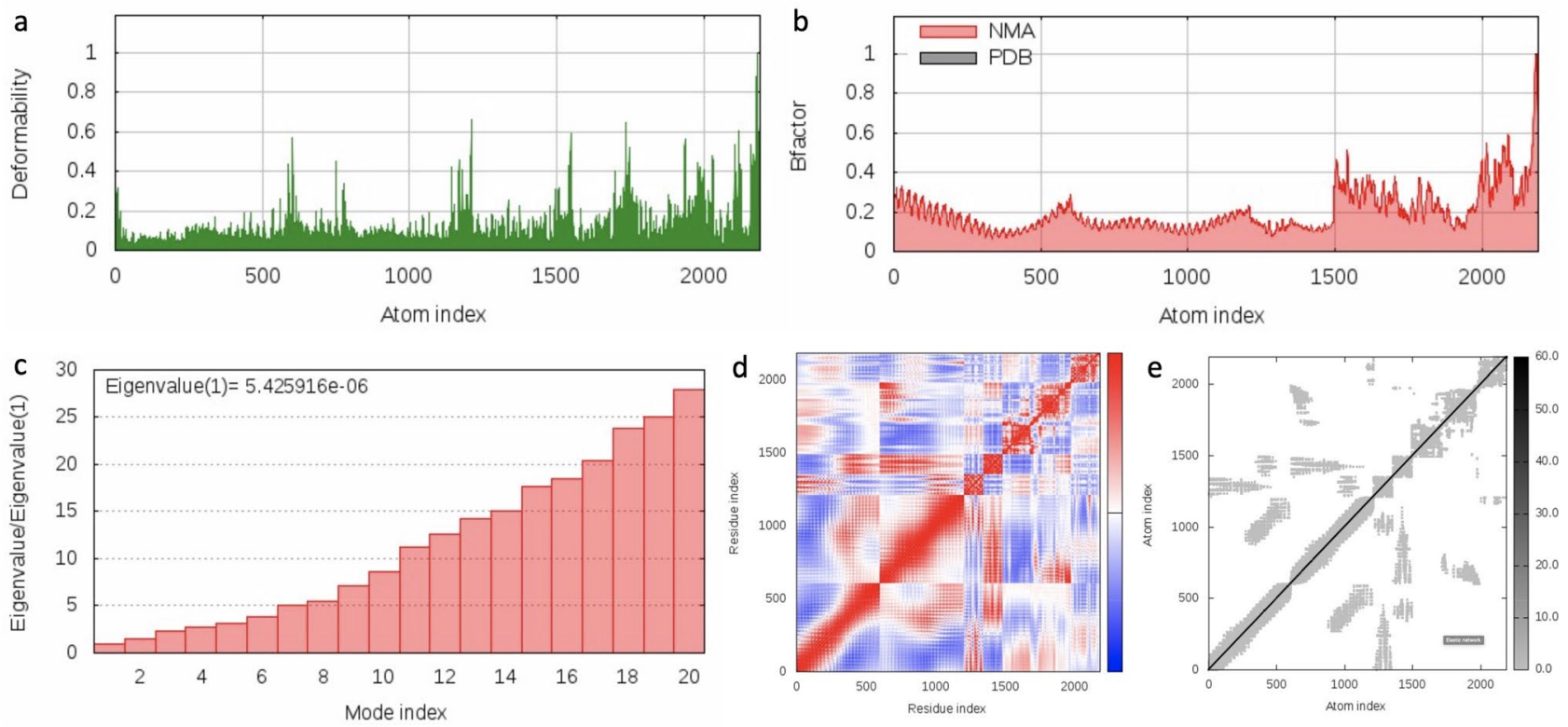

Figure 13. The molecular dynamics simulation of the vaccine-TLR4 docked complex. (a) Main-chain deformability simulation, the hinges are regions with high deformability. (b) B-factor values calculated by normal mode analysis, quantifying the uncertainty of each atom. (c) The eigenvalue of the docked complex, showing the energy required to deform the structure. (d) The covariance matrix between pairs of residues (red: correlated, white: uncorrelated, blue: anti-correlated). (e) The elastic network model, suggesting the connection between atoms and springs. The springs are more rigid if their greys are darker.

\begin{tabular}{|l|l|l|}
\hline Mutations & Occurrence & Regions \\
\hline G476S & 3 & Washington \\
\hline V483A & 6 & Washington \\
\hline D614G & 116 & Washington, Los Angeles, New York, South America, Europe \\
\hline
\end{tabular}

Table 12. Spike protein mutations. Occurrence is the number of isolates that showed the mutation. Region is the origin of the isolates.

In conclusion, our designed multi-epitope vaccine can tackle the current RNA mutations of the coronavirus. The current RNA mutations of the coronavirus create no new potential vaccine subunits.

\section{Discussion}

In silico vaccine design has high value of efficacy and it strongly emphasizes the multi-epitope in the vaccine peptides. In this study, we develop DeepVacPred, an efficient vaccine subunit sieving framework, that exploits an AI-based approach to rapidly select 26 potential vaccine subunit candidates, introducing a new way for achieving a much higher speed and efficiency in in silico vaccine design. The goal is to directly predict the potential vaccine subunit sequence without the need to do a large number of different predictions, as well as to evaluate and select the predicted results manually. With this AI-based framework, we are able to skip at least 95\% of unnecessary predictions and let the computer analyze and select the best vaccine subunits for us. DeepVacPred predicts the 26 vaccine subunits within less than a second, which enables us to skip the most time consuming part of the in silico vaccine design. With DeepVacPred, a researcher can construct a multi-epitope vaccine for a new virus and validate its quality within an hour.

This approach can be further developed by enhancing the complexity and coverage of the dataset. In this study, we selected a part of known epitopes and protective antigens to form the dataset and use it for training the DNN architecture. We use the simple bridging of one B-cell epitopes and one T-cell epitopes. With a more comprehensive dataset and more possibilities of epitope combinations, we will be able to develop a better, more comprehensive and quicker vaccine design tool. In spite of limited available datasets, the current framework can still deal with most of the situations now and provide an efficacious vaccine design.

The application of AI, and DNN methodology in particular, to protein sequences classification shows great potential. Most of the online tools rely on the SVM learning approaches. In the highly popular protective antigens prediction tool Vaxijen ${ }^{34}$, the AUC of the ROC curve can only reach 0.743 , which cannot perform very accurate predictions. The dataset to train Vaxijen only contains 200 proteins, so it becomes more time consuming and challenging to rely on the SVM model with increasing number of discovered protective antigens. Consequently, 
the proposed DeepVacPred proves that DNN can perform a very accurate prediction with over 700,000 different proteins in the dataset.

This study eventually results in a novel multi-epitope vaccine with a length of 649aa against the SARS-CoV-2. It contains an adjuvant, 11 subunits with $16 \mathrm{~B}$-cell epitopes, $82 \mathrm{CTL}$ epitopes and 89 HTL epitopes. It shows good antigenicity, population coverage and good physichochemical properties and structures, providing great potential for the next step COVID-19 vaccine design with actual experiments and clinical studies.

Furthermore, we trace the RNA mutations of the SARS-CoV-2 virus. Basically, the RNA mutations can result in one amino acid change in the spike protein or other related proteins. The proposed vaccine design framework can also tackle the three most frequently observed mutations as well as it can be extended to deal with other potentially unknown mutations. The investigation on the RNA mutations also proves the high efficiency of our DeepVacPred. As future work, we will investigate novel AI algorithms and architectures capable of constructing multi-epitope vaccine designs that can overcome the unknown unknowns of viruses evolution.

\section{Methods}

DNN design and training in DeepVacPred framework. Each data input to the DNN architecture is a sequence with a length of 45 vectors which is converted from its protein sequence by Z-descriptors ${ }^{31}$ and ACC transformation $^{32}$. Convolutional Neural Network $(\mathrm{CNN})$ exhibits good performance to identify and process such vectors while multi-layer linear neural network is broadly connected to the ouput layer of the CNN, forming a complex DNN to enhance the classification ability. Hence, our DNN is constructed by the following layers and the parameters of each layer is decided using a random search to obtain high accuracy while maintaining good computing speed:

i. $C N N$, in channels $=1$, out channels $=16$, kernel size $=3$, stride $=2$, padding $=1$, Tanh function;

ii. $C N N$, in channels $=16$, out channels $=16$, kernel size $=3$, stride $=2$, padding $=1$, Tanh function;

iii. $\mathrm{CNN}$, in channels $=16$, out channels $=1$, kernel size $=3$, stride $=2$, padding $=1$, Tanh function, average pooling;

iv. Linear, in features $=32$, out features $=64$, Tanh function;

v. Linear, in features $=64$, out features $=32$, Tanh function;

vi. Linear, in features $=32$, out features $=16$, Tanh function;

vii. Linear, in features $=16$, out features $=2$, Sigmoid function.

The hyper-parameters of the DNN training are listed below. The selected hyper-parameter values are marked in bold. We choose the hyper-parameters with good accuracy while maintaining good computing speed by using a random search.

i. Learning rate: $[0.0001,0.0005, \mathbf{0 . 0 0 1}, 0.0015,0.002]$;

ii. Optimizer: [SGD, RMSProp, Adam];

iii. Epochs: [2000, 4000, 6000, 8000, 10000];

iv. Batch size: [1024, 2048, 4096, 8192].

Linear B-cell epitopes prediction. We use four popular server to predict the linear B-cell epitopes on each vaccine subunit candidates. (1) BepiPred-2.0 web server (http://www.cbs.dtu.dk/services/BepiPred/). BepiPred is a reliable machine learning based tool trained by random forest algorithm and its training dataset covers a large number of known linear B-cell epitopes from the IEDB database ${ }^{25}$. (2) ABCpred (http://www. imtech.res.in/raghava/abcpred/). ABCPred applies recurrent neural network to the classification of epitopes and non-epitopes to improve the accuracy ${ }^{39}$. (3) SVMTrip (http://sysbio.unl.edu/SVMTriP/). SVMTrip uses support vector machine to predict antigenic epitopes and its AUC reaches a value of $0.702^{38}$. (4) BCPreds (http://ailab.ist. psu.edu/bcpred/). BCPreds is also based on SVM model with an AUC value of 0.758 and its prediction relies on kernel methods ${ }^{40}$. The B-cell surface accessibility is checked by IEDB Emini tool ${ }^{42}$.

Cytotoxic T lymphocytes (CTL) epitopes prediction. We use NetMHCpan 4.1 server (http://www.cbs. dtu.dk/services/NetMHCpan/) to predict the CTL epitopes on each vaccine subunit candidates. We predict the CTL epitopes with a length of 9aa. All the parameters are set at default. NetMHCpan predicts peptide binding to any MHC Class I molecule of known sequence using artificial neural networks (ANNs) which is trained on a combination of more than 850,000 quantitative Binding Affinity (BA) and Mass-Spectrometry Eluted Ligands (EL) peptides, providing reliable prediction results ${ }^{43}$.

Helper T lymphocytes (HTL) epitopes prediction. We use NetMHCIIpan 4.0 server (http://www.cbs. dtu.dk/services/NetMHCIIpan/) to predict the HTL epitopes on each vaccine subunit candidates. We predict the HTL epitopes with a length of $15 \mathrm{aa}$. All the parameters are set at default. NetMHCIIpan predicts peptide binding to any MHC II molecule of known sequence using artificial neural networks (ANNs) which is trained on an extensive dataset of over 500,000 measurements of Binding Affinity (BA) and Eluted Ligand mass spectrometry (EL), covering the three human HLA-DR, HLA-DQ and HLA-DP alleles, providing reliable prediction results ${ }^{46}$.

Multi-epitope vaccine construction. In this section, the BLASTp screening is done by the Uniprot server (https://www.uniprot.org/blast). BLASTp can identify similar regions between two sequences.

Worldwide human population coverage analysis. The worldwide human population coverage of each subunit is evaluated by IEDB population coverage analysis tool (http://tools.iedb.org/population/). The evluation is done on the worldwide human population. 
Antigenicity, allergenicity and solubility evaluation. The antigenicity of the final vaccine and its every subunit is predicted by VaxiJen 2.0 server (http://www.ddg-pharmfac.net/ vaxijen/VaxiJen/VaxiJen.html) and AntigenPro server (http://scratch.proteomics.ics.uci.edu). Vaxijen is based on auto cross covariance (ACC) transformation of protein sequences into uniform vectors of principal amino acid properties ${ }^{34}$. Antigenpro is a sequence-based, alignment-free and pathogen-independant predictor of protein antigenicity ${ }^{55}$. The allergenicity of the final vaccine and its every subunit is checked by AllergenFP 1.0 server (http://ddg-pharmfac.net/Aller genFP/) and AllerTOP 2.0 server (https://www.ddg-pharmfac.net/AllerTOP/). AllergenFP and is a binary classfier between allergens and non-allergens. The dataset is described by five E-descriptors and the strings are transformed into uniform vectors by auto-cross covariance (ACC) transformation ${ }^{76}$. AllerTop is also based on ACC transformation and E-descriptors ${ }^{56}$. The solubility is evaluated by SolPro server (http://scratch.proteomics.ics. uci.edu) and Protein-sol server (https://protein-sol.manchester.ac.uk). SolPro is an SVM based tool to predict the solubility of a protein sequence with an overall accuracy of over $74 \%$ estimated by tenfold cross-validation ${ }^{57}$. Protein-sol is based on available data for Escherichia coli protein solubility in a cell-free expression system ${ }^{58}$.

Toxicity and physicochemical properties analysis. The toxicity of the final vaccine and its every subunit is predicted by ToxinPred server (http://crdd.osdd.net/raghava/toxinpred/). TonxinPred is based on SVM model to classify toxicity and non-toxicity. The dataset used in its method consists of 1805 toxic peptides $(\leq 35$ residues $)^{60}$. The physicochemical properties of the final vaccine and its every subunit is predicted by ExPASy ProtParam server (https://web.expasy.org/protparam/). The physicochemical properties include hydropathicity, charge, half-life, instability index, pI (Theoretical isoelectric point value) and molecule wheight ${ }^{61}$.

Secondary structure prediction. PSIPRED is used for the secondary structure prediction of our final vaccine (http://bioinf.cs.ucl.ac.uk/psipred/). PSIPRED incorporates two feed-forward neural networks which perform an analysis on output obtained from PSI-BLAST (Position Specific Iterated-BLAST). It achieves an average Q3 score of $81.6 \%$, which can achieve accurate secondary structure prediction ${ }^{63}$. We also use RaptorX Property web server (http://raptorx.uchicago.edu/StructurePropertyPred/predict/) to predict the solvent accessibility (ACC) and disorder regions (DISO). RaptorX employs an emerging machine learning model called DeepCNF (Deep Convolutional Neural Fields) to predict secondary structure (SS), solvent accessibility (ACC), and disorder regions (DISO) simultaneously ${ }^{65}$.

Vaccine 3D structure modeling. The 3D model of the final vaccine is constructed by RaptorX server (http://raptorx.uchicago.edu/ContactMap). RaptorX provides distance-based protein folding powered by deep learning. This server was officially ranked 1st in contact prediction in both CASP12 and CASP13 and initiated the revolution of protein structure prediction by deep learning ${ }^{66}$.

Vaccine 3D structure refinement. The 3D model built by RaptorX server is refined by GalaxyRefine (http://galaxy.seoklab.org/cgi-bin/submit.cgi?type=REFINE). GalaxyRefine first rebuilds side chains and performs side-chain repacking and subsequent overall structure relaxation by molecular dynamics simulation. According to the CASP10 assessment, the GalaxyRefine server method performed the best in improving local structure quality ${ }^{67}$ The quality of the refined model is evaluated in terms of its GDT-HA socre, RMSD score, Molprobity score, clash score and Ramachandran plot score.

Vaccine 3D structure validation. The final refined 3D model of our final vaccine is validated by ProSAweb server(https://prosa.services.came.sbg.ac.at/prosa.php). ProSA calculates an overall quality score for a specific input structure. If this score is outside a range characteristic for native proteins the structure probably contains errors. A plot of local quality scores points to problematic parts of the model which are also highlighted in a $3 \mathrm{D}$ molecule viewer to facilitate their detection ${ }^{68}$.

Conformational B-cell epitope prediction. The conformational B-cell epitopes in the refined final vaccine 3D structure model are predicted by the ElliPro Server (http: //tools.iedb.org/ellipro). ElliPro is based on the geometrical properties of protein structure. Among the current conformational B-cell epitope prediction tools, ElliPro has the best AUC score of 0.732 , which is a very reliable tool for identifying antibody epitopes in protein antigens ${ }^{69}$.

Codon optimization and in silico cloning. Java Codon Adaptation Tool (JCat) server is used for codon optimization (https://urldefense.com/v3/_http://www.jcat.de/LIr3w8kk_Xxm!7wRJ08pRiYapODc_10a3L u91JwL-k63K5zWwthwiCfq_ctg6SmoWSkB2JxUzyRA). JCat adapts the codon usage to most sequenced prokaryotic organisms and selected eukaryotic organisms ${ }^{70}$. The optimized codon sequence is insert into pET28a $(+)$ vector with SnapGene 5.1.5 software (from Insightful Science; available at https://www.snapgene.com).

Molecular docking. The molecular docking is done by ClusPro 2.0 server (https://cluspro.bu.edu). ClusPro is a widely used tool for protein-protein docking. Docking with each energy parameter set results in ten models defined by centers of highly populated clusters of low-energy docked structures ${ }^{72}$. We choose TLR4 (PDB ID: $4 \mathrm{G} 8 \mathrm{~A}$ ) as the immune receptor. We select the docked complex with the lowest energy score.

Molecular dynamics simulation of the vaccine-receptor complex. The molecular dynamics simulation is done by iMOD server (iMODS) (http://imods.chaconlab.org). iMODS facilitates the exploration of 
such modes and generates feasible transition pathways between two homologous structures ${ }^{74}$. The iMOD server evaluates the protein stability by computing its internal coordinates through normal mode analysis (NMA). The stability of the protein is represented in terms of its main-chain deformability plot, B-factor values, eigenvalue, covariance matrix and elastic network model.

\section{Data availability}

We obtained the genome sequence and the spike protein sequence of SARS-CoV-2 from NCBI database (https ://www.ncbi.nlm. nih.gov) with accession number MN908947 and protein ID QHD43416.1. The protein data we collected and processed to train the DeepVacPred is available on github.com (https:/github.com/zikunyang/ DCVST).

\section{Code availability}

The code used for data generation and/or analysis in the study are available on github.com (https://github.com/ zikunyang/DCVST).

Received: 17 June 2020; Accepted: 11 January 2021

Published online: 05 February 2021

\section{References}

1. Wu, J., Leung, K. \& Leung, G. Nowcasting and forecasting the potential domestic and international spread of the 2019-nCoV outbreak originating in Wuhan, China: A modelling study. Lancet 395, 689-697. https://doi.org/10.1016/S0140-6736(20)30260-9 (2020).

2. Zhou, P. et al. A pneumonia outbreak associated with a new coronavirus of probable bat origin. Nature 579, 270-273. https://doi. org/10.1038/s41586-020-2012-7 (2020).

3. Dong, E., Du, H. \& Gardner, L. An interactive web-based dashboard to track COVID-19 in real time. Lancet 20, 533-534. https ://doi.org/10.1016/S1473-3099(20)30120-1 (2020).

4. Coronavirus: The first three months as it happened. Nature. https://doi.org/10.1038/d41586-020-00154-w (2020).

5. Shang, W. et al. The outbreak of SARS-CoV-2 pneumonia calls for viral vaccines. npj Vaccines. https://doi.org/10.1038/s4154 1-020-0170-0 (2020).

6. Tay, M. Z. et al. The trinity of COVID-19: Immunity, inflammation and intervention. Nat. Rev. Immunol. 20, 363-374. https://doi. org/10.1038/s41577-020-0311-8 (2020).

7. Huang, C. et al. Clinical features of patients infected with 2019 novel coronavirus in Wuhan, China. Lancet 395, 497-506. https:// doi.org/10.1016/S0140-6736(20)30183-5 (2020).

8. Chen, Y., Liu, Q. \& Guo, D. Emerging coronaviruses: Genome structure, replication, and pathogenesis. J. Med. Virol. 92, 418-423. https://doi.org/10.1002/jmv.25681 (2020).

9. Gewin, V. On the front lines of the coronavirus-vaccine battle. Nature https://doi.org/10.1038/d41586-020-01116-y (2020).

10. Callaway, E. The race for coronavirus vaccines: A graphical guide. Nature 580, 576-577. https://doi.org/10.1038/d41586-020-01221 -y (2020).

11. Graham, B. Advances in antiviral vaccine development. Immunol. Rev. 255, 230-242. https://doi.org/10.1111/imr.12098 (2013).

12. Gandon, S., Mackinnon, M., Nee, S. \& Read, F. Imperfect vaccines and the evolution of pathogen virulence. Nature 414, 751-756. https://doi.org/10.1038/414751a (2001).

13. Gao, Q. et al. Rapid development of an inactivated vaccine for SARS-CoV-2. BioRxiv, https://doi.org/10.1101/2020.04.17.046375 (2020).

14. Kim, Y. C., Dema, B. \& Reyes-Sandoval, A. COVID-19 vaccines: Breaking record times to first-in-human trials. npj Vaccines. https ://doi.org/10.1038/s41541-020-0188-3 (2020).

15. Oany, A., Emran, A. \& Jyoti, T. Design of an epitope-based peptide vaccine against spike protein of human coronavirus: An in silico approach. Drug Des. Dev. Ther. 8, 1139-1149. https://doi.org/10.2147/DDDT.S67861 (2014).

16. Feng, Y., Qiu, M., Zou, S. et al. Multi-epitope vaccine design using an immunoinformatics approach for 2019 novel coronavirus in China (SARS-CoV-2). BioRxiv, https://doi.org/10.1101/2020.03.03.962332 (2020).

17. Samad, A. et al. Designing a multi-epitope vaccine against SARS-CoV-2: An immunoinformatics approach. J. Biomol. Struct. Dyn. https://doi.org/10.1080/07391102.2020.1792347 (2020).

18. Zhang, L. Multi-epitope vaccines: A promising strategy against tumors and viral infections. Cell Mol Immunol 15, 182-184. https ://doi.org/10.1038/cmi.2017.92 (2017).

19. Lan, J. et al. Structure of the SARS-CoV-2 spike receptor-binding domain bound to the ACE2 receptor. Nature 581, 215-220. https ://doi.org/10.1038/s41586-020-2180-5 (2020).

20. Lu, R. et al. Genomic characterisation and epidemiology of 2019 novel coronavirus: Implications for virus origins and receptor binding. Lancet 395, 565-574. https://doi.org/10.1016/S0140-6736(20)30251-8 (2020).

21. Shokeen, K., Pandey, S., Shah, M. \& Kumar, S. Insight towards the effect of the multibasic cleavage site of SARS-CoV-2 spike protein on cellular proteases. BioRxiv https://doi.org/10.1101/2020.04.25.061507 (2020).

22. Purcell, A., McCluskey, J. \& Rossjohn, J. More than one reason to rethink the use of peptides in vaccine design. Nat. Rev. Drug Discov. 6, 404-414. https://doi.org/10.1038/nrd2224 (2007).

23. Callaway, E. Scores of coronavirus vaccines are in competition-How will scientists choose the best?. Nature https://doi. org/10.1038/d41586-020-01247-2 (2020).

24. Mascola, J. R. \& Fauci, A. S. Novel vaccine technologies for the 21 st century. Nat. Rev. Immunol. 20, 87-88. https://doi.org/10.1038/ s41577-019-0243-3 (2020).

25. Jespersen, M., Peters, B., Nielsen, M. \& Marcatili, P. BepiPred-2.0: Improving sequence-based B-cell epitope prediction using conformational epitopes. Nucleic Acids Res. 45, 24-29. https://doi.org/10.1093/nar/gkx346 (2017).

26. Nielsen, M. et al. NetMHCpan, a method for quantitative predictions of peptide binding to any HLA-A and -B locus protein of known sequence. PLoS ONE 2, e796. https://doi.org/10.1371/journal.pone.0000796 (2007).

27. Zhu, X. \& Goldberg, A. Introduction to semi-supervised learning. Morgan Claypool Publ. https://doi.org/10.2200/S00196ED1V 01Y200906AIM006 (2009).

28. Ahmad, T., Eweida, A. \& El-Sayed, L. T-cell epitope mapping for the design of powerful vaccines. Anal. Chim Acta 6, 13-22. https ://doi.org/10.1016/j.vacrep.2016.07.002 (2016).

29. Heinson, A. et al. Enhancing the biological relevance of machine learning classifiers for reverse vaccinology. Int. J. Mol. Sci. 18, 312. https://doi.org/10.3390/ijms18020312 (2017).

30. Agesen, O. The Cartesian product algorithm. 9th Eur. Conf. https://doi.org/10.1007/3-540-49538-X_2 (1995). 
31. Hellberg, S., Sjoestroem, M., Skagerberg, B. \& Wold, S. Peptide quantitative structure-activity relationships, a multivariate approach. Am. Chem. Soc. 30, 1126-1135. https://doi.org/10.1021/jm00390a003 (1987).

32. Wold, S., Jonsson, J., Sjöström, M., Sandberg, M. \& Rännar, S. DNA and peptide sequences and chemical processes multivariately modeled by principal component analysis and partial least squares projections to latent structures. Anal Chim Acta 277, 239-253. https://doi.org/10.1016/0003-2670(93)80437-P (1993).

33. Calders, T. \& Jaroszewicz, S. Efficient AUC optimization for classification. Knowl. Discov. Databases https://doi.org/10.1007/9783-540-74976-9_8 (2007).

34. Doytchinova, I. A. \& Flower, D. R. VaxiJen: A server for prediction of protective antigens, tumour antigens and subunit vaccines. BMC Bioinform. https://doi.org/10.1186/1471-2105-8-4 (2007).

35. Wu, F. et al. A new coronavirus associated with human respiratory disease in China. Nature 579, 265-269. https://doi.org/10.1038/ s41586-020-2008-3 (2020).

36. Patronov, A. \& Doytchinova, I. T-cell epitope vaccine design by immunoinformatics. Open Biol. https://doi.org/10.1098/rsob.12013 9 (2013).

37. Sanchez-Trincado, J., Gomez-Perosanz, M. \& Reche, P. Fundamentals and methods for T- and B-cell epitope prediction. J. Immunol. Res. https://doi.org/10.1155/2017/2680160 (2017).

38. Yao, B., Zhang, L., Liang, S. \& Zhang, C. SVMTriP: A method to predict antigenic epitopes using support vector machine to integrate tri-peptide similarity and propensity. PLoS ONE 7, 45152. https://doi.org/10.1371/journal.pone.0045152 (2012).

39. Saha, S. \& Raghava, G. P. S. Prediction of continuous B-cell epitopes in an antigen using recurrent neural network. Proteins 65, 40-48. https://doi.org/10.1002/prot.21078 (2006).

40. El-Manzalawy, Y., Dobbs, D. \& Honavar, V. Predicting linear B-cell epitopes using string kernels. J. Mol. Recognit. 21, $243-255$. https://doi.org/10.1002/jmr.893 (2008).

41. Almofti, Y., Abd-elrahman, K., Gassmallah, S. \& Salih, M. Multi epitopes vaccine prediction against severe acute respiratory syndrome (SARS) coronavirus using immunoinformatics approaches. Am. J. Microbiol. Res. 6, 94-114, https://doi.org/10.12691/ ajmr-6-3-5 (2018)

42. Emini, E. A., Hughes, J. V., Perlow, D. S. \& Boger, J. Induction of hepatitis A virus-neutralizing antibody by a virus-specific synthetic peptide. J. Virol. 55, 836-839. https://doi.org/10.1128/JVI.55.3.836-839.1985 (1985)

43. Jurtz, V. et al. NetMHCpan-40: Improved peptide-MHC class I interaction predictions integrating eluted ligand and peptide binding affinity data. J. Immunol. 199, 3360-3368. https://doi.org/10.4049/jimmunol.1700893 (2017).

44. Robinson, J. et al. IMGT/HLA-A sequence database for the human major histocompatibility complex. Tissue Antigens 55, $280-287$. https://doi.org/10.1128/JVI.55.3.836-839.1985 (2000).

45. Nielsen, M. et al. Quantitative predictions of peptide binding to any HLA-DR molecule of known sequence: NetMHCIIpan. PLoS Comput. Biol. https://doi.org/10.1371/journal.pcbi.1000107 (2008).

46. Reynisson, B. et al. Improved prediction of MHC II antigen presentation through integration and motif deconvolution of mass spectrometry MHC eluted ligand data. J. Proteome Res. https://doi.org/10.1021/acs.jproteome.9b00874 (2020).

47. Reynisson, B. et al. Improved prediction of MHC II antigen presentation through integration and motif deconvolution of mass spectrometry MHC eluted ligand data. J. Proteome Res. https://doi.org/10.1021/acs.jproteome.9b00874 (2020).

48. Bui, H. H. et al. Predicting population coverage of T-cell epitope-based diagnostics and vaccines. BMC Bioinform. https://doi. org/10.1186/1471-2105-7-153 (2006).

49. Man, L., Jiang, Y., Gong, T., Zhang, Z. \& Sun, X. Intranasal vaccination against HIV-1 with adenoviral vector based nanocomplex using synthetic TLR-4 agonist peptide as adjuvant. Mol. Pharm. 13, 885-894. https://doi.org/10.1021/acs.molpharmaceut.5b008 02 (2016).

50. Diedrich, G. et al. Ribosomal protein L2 is involved in the association of the ribosomal subunits, tRNA binding to A and P sites and peptidyl transfer. EMBO J. 19, 5241-5250. https://doi.org/10.1093/emboj/19.19.5241 (2000).

51. Singh, M. \& O'Hagan, D. Advances in vaccine adjuvants. Nat. Biotechnol. 17, 1075-1081. https://doi.org/10.1038/15058 (1999).

52. Arai, R., Ueda, H., Kitayama, A., Kamiya, N. \& Nagamune, T. Design of the linkers which effectively separate domains of a bifunctional fusion protein. Protein Eng. Des. Sel. 14, 529-532. https://doi.org/10.1093/protein/14.8.529 (2001).

53. Crowe, J., Masone, B. S. \& Ribbe, J. One-step purification of recombinant proteins with the 6xHis tag and Ni-NTA resin. Mol. Biotechnol. 4, 247-258. https://doi.org/10.1007/BF02779018 (1995).

54. Ong, E. et al. Vaxign-ML: Supervised machine learning reverse vaccinology model for improved prediction of bacterial protective antigens. Bioinformatics 36, 3185-3191. https://doi.org/10.1093/bioinformatics/btaa119 (2020).

55. Magnan, C. et al. High-throughput prediction of protein antigenicity using protein microarray data. Bioinformatics 26, $2936-2943$. https://doi.org/10.1093/bioinformatics/btq551 (2010).

56. Dimitrov, I., Flower, D. R. \& Doytchinova, I. AllerTOP-A server for in silico prediction of allergens. Bioinformatics 14, S4. https ://doi.org/10.1186/1471-2105-14-S6-S4 (2013)

57. Dimitrov, I., Randall, A. \& Baldi, P. SOLpro: Accurate sequence-based prediction of protein solubility. Bioinformatics 25, 2200-2207. https://doi.org/10.1186/1471-2105-14-S6-S4 (2009).

58. Hebditch, M. et al. Protein-Sol: A web tool for predicting protein solubility from sequence. Bioinformatics 33, 3098-3100. https ://doi.org/10.1093/bioinformatics/btx345 (2017).

59. Iwasaki, A. \& Yang, Y. The potential danger of suboptimal antibody responses in COVID-19. Nat. Rev. Immunol. https://doi. org/10.1038/s41577-020-0321-6 (2020).

60. Gupta, S. et al. In silico approach for predicting toxicity of peptides and proteins. PLoS ONE 8, e73597. https://doi.org/10.1371/ journal.pone.0073957 (2013).

61. Gasteiger, E. et al. John M. Walker: Protein identification and analysis tools on the ExPASy server. Proteomics Protoc. Handb. https ://doi.org/10.1385/1592598900 (2005).

62. Pandey, A. M. et al. Exploring dengue genome to construct a multi-epitope based subunit vaccine by utilizing immunoinformatics approach to battle against dengue infection. Sci. Rep. https://doi.org/10.1038/s41598-017-09199-w (2017).

63. McGuffin, L. J., Bryson, K. \& Jones, D. T. The PSIPRED protein structure prediction server. Bioinformatics 16, 1511-1522. https ://doi.org/10.1093/bioinformatics/16.4.404 (2000).

64. Källberg, M. et al. Template-based protein structure modeling using the RaptorX web server. Nat. Protoc. 7, 1511-1522. https:// doi.org/10.1038/nprot.2012.085 (2012)

65. Wang, S., Li, W., Liu, S. \& Xu, J. RaptorX-property: A web server for protein structure property prediction. Nucleic Acids Res. 44, W430-W435. https://doi.org/10.1093/nar/gkw306 (2016).

66. Wang, S., Li, W., Zhang, R., Liu, S. \& Xu, J. CoinFold: A web server for protein contact prediction and contact-assisted protein folding. Nucleic Acids Res. 44, W361-W366. https://doi.org/10.1093/nar/gkw307 (2016).

67. Heo, L., Park, H. \& Seok, C. GalaxyRefine: Protein structure refinement driven by side-chain repacking. Nucleic Acids Res. 41, W384-W388. https://doi.org/10.1093/nar/gkt458 (2013).

68. Wiederstein, M. \& Sippl, M. J. ProSA-web: Interactive web service for the recognition of errors in three-dimensional structures of proteins. Nucleic Acids Res. 35, W407-W410. https://doi.org/10.1093/nar/gkm290 (2007).

69. Ponomarenko, J. V. et al. ElliPro: A new structure-based tool for the prediction of antibody epitopes. BMC Bioinform. https://doi. org/10.1186/1471-2105-9-514 (2008). 
70. Grote, A. et al. JCat: A novel tool to adapt codon usage of a target gene to its potential expression host. Nucleic Acids Res. 33, W526-W531. https://doi.org/10.1093/nar/gki376 (2005).

71. Nain, Z., Karim, M. M., Sen, M. K. \& Adhikari, U. K. Structural basis and designing of peptide vaccine using PE-PGRS family protein of Mycobacterium ulcerans-An integrated vaccinomics approach. Mol. Immunol. 120, 146-163. https://doi.org/10.1016/j. molimm.2020.02.009 (2020).

72. Kozakov, D. et al. The ClusPro web server for protein-protein docking. Nat. Protoc. 12, 255-278. https://doi.org/10.1038/nprot .2016.169 (2017).

73. Sayed, S. B. et al. Exploring lassa virus proteome to design a multi-epitope vaccine through immunoinformatics and immune simulation analyses. Int. J. Pept. Res. Ther. https://doi.org/10.1007/s10989-019-10003-8 (2020).

74. López-Blanco, J. R., Aliaga, J., Quintana-Ortí, E. S. \& Chacón, P. iMODS: Internal coordinates normal mode analysis server. Nucleic Acids Res. 42, W271-W276. https://doi.org/10.1093/nar/gku339 (2014).

75. Banerjee, A. K., Begum, F. \& Ray, U. Mutation hot spots in spike protein of COVID-19. Preprints 2020, 2020040281, https://doi. org/10.20944/preprints202004.0281.v1 (2020).

76. Ivan, D. et al. AllergenFP: Allergenicity prediction by descriptor fingerprints. Bioinformatics 6, 846-851. https://doi.org/10.1093/ bioinformatics/btt619 (2014).

\section{Acknowledgements}

The authors gratefully acknowledge the support by the National Science Foundation under the Career Award CPS/CNS-1453860, the NSF award under Grant numbers CCF-1837131, MCB-1936775, and CNS-1932620, the U.S. Army Research Office (ARO) under Grant No. W911NF-17-1-0076 and the DARPA Young Faculty Award and DARPA Director Award, under grant number N66001-17-1-4044, and a Northrop Grumman grant. The views, opinions, and/or findings contained in this article are those of the authors and should not be interpreted as representing the official views or policies, either expressed or implied by the Defense Advanced Research Projects Agency, the Department of Defense or the National Science Foundation.

\section{Author contributions}

Z.Y., P.B. and S.N. conceived the problem formulation and discussed the computational approach as well as the experimental methodology. Z.Y. designed, implemented, improved analyzed the experimental results. All authors analyzed the results and improved the manuscript.

\section{Competing interests}

The authors declare no competing interests.

\section{Additional information}

Correspondence and requests for materials should be addressed to P.B.

Reprints and permissions information is available at www.nature.com/reprints.

Publisher's note Springer Nature remains neutral with regard to jurisdictional claims in published maps and institutional affiliations.

(c) (i) Open Access This article is licensed under a Creative Commons Attribution 4.0 International (c) License, which permits use, sharing, adaptation, distribution and reproduction in any medium or format, as long as you give appropriate credit to the original author(s) and the source, provide a link to the Creative Commons licence, and indicate if changes were made. The images or other third party material in this article are included in the article's Creative Commons licence, unless indicated otherwise in a credit line to the material. If material is not included in the article's Creative Commons licence and your intended use is not permitted by statutory regulation or exceeds the permitted use, you will need to obtain permission directly from the copyright holder. To view a copy of this licence, visit http://creativecommons.org/licenses/by/4.0/.

(C) The Author(s) 2021 